\title{
Intravenously injected human multilineage-differentiating stress-enduring cells selectively engraft into mouse aortic aneurysms and attenuate dilatation by differentiating into multiple cell types
}

\author{
Katsuhiro Hosoyama, MD, PhD, ${ }^{a, b}$ Shohei Wakao, PhD, ${ }^{b}$ Yoshihiro Kushida, PhD, ${ }^{b}$ Fumitaka Ogura, PhD, \\ Kay Maeda, MD, PhD, ${ }^{a}$ Osamu Adachi, MD, PhD, ${ }^{a}$ Shunsuke Kawamoto, MD, PhD, ${ }^{a}$ \\ Mari Dezawa, MD, $\mathrm{PhD},{ }^{\mathrm{b}}$ and Yoshikatsu Saiki, $\mathrm{MD}, \mathrm{PhD}^{\mathrm{a}}$
}

\section{ABSTRACT}

Objectives: Aortic aneurysms result from the degradation of multiple components represented by endothelial cells, vascular smooth muscle cells, and elastic fibers. Cells that can replenish these components are desirable for cell-based therapy. Intravenously injected multilineage-differentiating stress-enduring (Muse) cells, endogenous nontumorigenic pluripotent-like stem cells, reportedly integrate into the damaged site and repair the tissue through spontaneous differentiation into tissue-compatible cells. We evaluated the therapeutic efficacy of Muse cells in a murine aortic aneurysm model.

Methods: Human bone marrow Muse cells, isolated as stage-specific embryonic antigen $-3^{+}$from bone marrow mesenchymal stem cells, or non-Muse cells (stagespecific embryonic antigen- $3^{-}$cells in mesenchymal stem cells), bone marrow mesenchymal stem cells, or vehicle was intravenously injected at day 0 , day 7 , and 2 weeks (20,000 cells/injection) after inducing aortic aneurysms by periaortic incubation of $\mathrm{CaCl}_{2}$ and elastase in severe combined immunodeficient mice.

Results: At 8 weeks, infusion of human Muse cells attenuated aneurysm dilation, and the aneurysmal size in the Muse group corresponded to approximately $62.5 \%, 55.6 \%$, and $45.6 \%$ in the non-Muse, mesenchymal stem cell, and vehicle groups, respectively. Multiphoton laser confocal microscopy revealed that infused Muse cells migrated into aneurysmal tissue from the adventitial side and penetrated toward the luminal side. Histologic analysis demonstrated robust preservation of elastic fibers and spontaneous differentiation into endothelial cells and vascular smooth muscle cells.

Conclusions: After intravenous injection, Muse cells homed and expanded to the aneurysm from the adventitial side. Subsequently, Muse cells differentiated spontaneously into vascular smooth muscle cells and endothelial cells, and elastic fibers were preserved. These Muse cell features together led to substantial attenuation of aneurysmal dilation. (J Thorac Cardiovasc Surg 2018;155:2301-13)

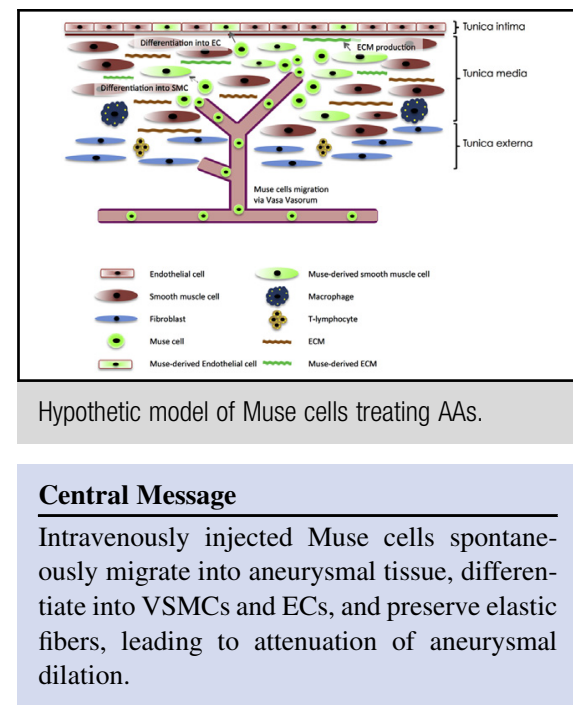

Perspective

After intravenous injection, Muse cells homed into the AA and expanded to the aortic tissue. After integration, Muse cells differentiated spontaneously into VSMCs and ECs, and elastic fibers were preserved. These Muse cell features together led to substantial attenuation of AA dilation. We believe that Muse cells may be viable options for cell therapies.

See Editorial Commentary page 2314
The aorta is a well-organized, multilayered structure comprising several cell types, namely, endothelial cells (ECs), vascular smooth muscle cells (VSMCs), and

\footnotetext{
From the ${ }^{\mathrm{a}}$ Division of Cardiovascular Surgery and ${ }^{\mathrm{b}}$ Department of Stem Cell Biology and Histology, Tohoku University Graduate School of Medicine, Sendai, Miyagi, Japan.

Funded by the Japan Society for the Promotion of Science (JSPS) KAKENHI Grant (JP26462097).

Received for publication Aug 3, 2017; revisions received Jan 13, 2018; accepted for publication Jan 22, 2018; available ahead of print March 17, 2018.

Address for reprints: Katsuhiro Hosoyama, MD, PhD, or Yoshikatsu Saiki, MD, PhD, Division of Cardiovascular Surgery, Tohoku University Graduate School of Med-
}

fibroblasts, as well as an extracellular matrix (ECM), which includes elastic and collagen fibers. Aortic aneurysms (AAs) are defined as progressive enlargements of the aorta

icine, 1-1 Seiryo-cho, Aoba-ku, Sendai, Miyagi 980-8574, Japan (E-mail: k hosoyama@med.tohoku.ac.jp or yoshisaiki@med.tohoku.ac.jp); or Mari Dezawa, $\mathrm{MD}, \mathrm{PhD}$, Department of Stem Cell Biology and Histology, Tohoku University Graduate School of Medicine, 2-1 Seiryo-cho, Aoba-ku, Sendai, Miyagi 980-8575, Japan (E-mail: mdezawa@med.tohoku.ac.jp). $0022-5223 / \$ 36.00$

Copyright $(2018$ by The American Association for Thoracic Surgery

https://doi.org/10.1016/j.jtcvs.2018.01.098 


$\begin{array}{ll}\text { Abbreviations and Acronyms } \\ \text { AA } & =\text { aortic aneurysm } \\ \alpha \text { SMA } & =\alpha \text { smooth muscle actin } \\ \text { BM } & =\text { bone marrow } \\ \text { CXCR } 4 & =\text { C-X-C chemokine receptor type } 4 \\ \text { EC } & =\text { endothelial cell } \\ \text { ECM } & =\text { extracellular matrix } \\ \text { EPC } & =\text { endothelial progenitor cell } \\ \text { GFP } & =\text { green fluorescent protein } \\ \text { Ig } & =\text { immunoglobulin } \\ \text { MSC } & =\text { mesenchymal stem cell } \\ \text { Muse cell } & =\text { multilineage-differentiating stress- } \\ & \text { enduring cell } \\ \text { PBS } & =\text { phosphate-buffered saline } \\ \text { SCID mouse } & =\text { severe combined immunodeficient } \\ & \text { mouse } \\ \text { SSEA-3 } & =\text { stage-specific embryonic antigen-3 } \\ \text { VSMC } & =\text { vascular smooth muscle cell }\end{array}$

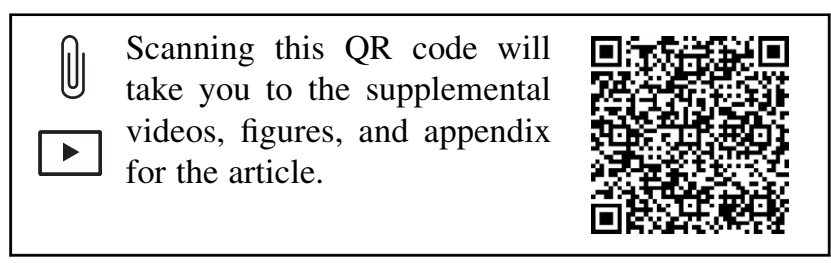

caused by multistep destruction of the tissue; atherosclerosis, inflammation, and oxidative stress cause the infiltration of lymphocytes and monocytes/macrophages, leading to the activation of matrix metalloproteinases, and inflammatory cytokines and chemokines. ${ }^{1-6}$ This series of reactions converges to thin the aortic wall because of the loss of the structural backbone of VSMCs, ECM, and ECs, which eventually results in an irreversible aneurysmal dilation that carries an incremental risk of rupture as the diameter increases over time. ${ }^{5}$ To date, cellbased therapy has been applied to animal models using several types of cells, such as VSMCs, ${ }^{7-9} \mathrm{ECs},{ }^{10}$ and mesenchymal stem cells (MSCs), including adipose-derived stem cells ${ }^{11-17}$ and skeletal muscle-derived stem cells. ${ }^{18}$ These cells deliver beneficial outcomes for AAs, particularly by paracrine and immunomodulatory effects, whereas the attenuation and inhibition of aneurysmal dilation with a robust tissue repair are insufficient. ${ }^{11-17}$

Multilineage-differentiating stress-enduring (Muse) cells were first reported by Kuroda and colleagues in $2010,{ }^{19}$ as endogenous nontumorigenic pluripotent-like stem cells that reside in the connective tissue of nearly every organ and in the bone marrow (BM) and peripheral blood. ${ }^{20}$ Muse cells may be collected as cells positive for the pluripotent surface marker stage-specific embryonic antigen
(SSEA)-3, which is expressed on pluripotent cells in normal development in the fertilized egg to epiblast stem cells, as well as on human embryonic stem cells. ${ }^{21}$ Muse cells are also included as several percent of commercially available cultured mesenchymal cells, such as fibroblasts and BMMSCs. Consistent with the fact that Muse cells reside in normal tissue, they are nontumorigenic; their telomerase activity is at the somatic cell level, and these cells do not exhibit an abnormal karyotype after several passages, nor do they form tumors after transplantation in vivo. ${ }^{21}$ The doubling time of Muse cells is 1.3 days/cell division, suggesting their feasibility for therapeutic application. ${ }^{22}$

Because Muse cells are pluripotent-like, they have the ability to differentiate into cells representative of all 3 germ layers from a single cell and to self-renew. Moreover, as demonstrated in liver- and skeletal muscle-damaged models, Muse cells home to the site of damage after simple intravenous injection and repair the tissue by replenishing new functional cells through spontaneous differentiation into tissue-compatible cells. ${ }^{19,23}$ In addition, Muse cells secrete factors that enable them to survive under stressful conditions and to suppress immunologic reactions. ${ }^{22,24}$

Because of these unique properties, Muse cells are expected to provide an efficient therapeutic efficacy for AA by simple intravenous injection because of their stress tolerance, which enables them to survive in the destructive AA tissue; their ability to selectively home to the damaged site; and their tissue reparative capability through spontaneous differentiation into multiple cell types that comprise the aorta. This study aimed to elucidate the efficacy, safety, and applicability of intravenously injected human Muse cells in a murine AA model.

\section{MATERIALS AND METHODS}

Detailed methods are provided in the Supplemental Methods. All animal experiments were approved by the Tohoku University (Sendai, Japan) Animal Care and Use Committee (No. 2013-543-1).

\section{Muse Cell Preparation and Characterization}

Human BM-MSCs were labeled with green fluorescent protein (GFP)labeled lentivirus as previously described. ${ }^{25}$ With the use of a fluorescenceactivated cell sorter (Aria II, Becton Dickinson, Franklin Lakes, NJ), Muse cells were collected as the $\mathrm{GFP}^{+} /$stage-specific embryonic antigen-3 $(\mathrm{SSEA}-3)^{+}$cell fraction (Figure E1, P3 gate), and non-Muse cells were collected as $\mathrm{GFP}^{+} / \mathrm{SSEA}^{-} 3^{-}$cell fraction (Figure E1, P6 gate). GFP ${ }^{+}$ MSCs were also sorted and used for the MSC experimental group.

\section{Induction of Aortic Aneurysm}

Eight-week-old male severe combined immunodeficient (SCID) mice (C.B-17/lcr-scid/scidJcl) were purchased from CLEA Japan (Tokyo, Japan). After isolation of the aortic segment, infrarenal to the iliac bifurcation, with ligation of lumbar arteries with 11-0 nylon (Kono Seisakusho, Tokyo, Japan), experimental models of abdominal AA were developed by 20 -minute periaortic incubation of $\mathrm{CaCl}_{2}(0.5 \mathrm{~mol} / \mathrm{L}$; Otsuka, Tokyo, Japan) and porcine pancreatic elastase $(0.5 \mathrm{unit} / \mu \mathrm{L}$, Wako Pure Chemical Industries, Osaka, Japan), as previously described. ${ }^{26}$ On week 3 or week 8 , 
after intracardial perfusion with $4 \%$ paraformaldehyde, the abdominal aortas were harvested, embedded in optimal cutting temperature compound, and subjected to further histologic analysis.

\section{Aortic Aneurysm Co-Culture With Muse Cells \\ Longitudinal murine AA tissue was dissected out, and then 10,000 $\mathrm{GFP}^{+}$-Muse cells were added onto the luminal side of the AA, the side on which intravenously injected Muse cells appear to interact directly in vivo. The AA samples were inspected with multiphoton laser micro- scope and subjected to further histologic assessment.}

\section{Cell Transplantation}

The AA exhibited greater than 1.5 times dilation based on ultrasound inspection 3 days after the initial induction, and the mice were divided randomly into 5 groups receiving (1) Muse cells (M group, $\mathrm{n}=16$ ), (2) single injection of Muse cells (M' group, $\mathrm{n}=16$ ), (3) non-Muse cells ( $\mathrm{N}$ group, $\mathrm{n}=16$ ), (4) MSCs (MSC group, $\mathrm{n}=16$ ), or (5) phosphate-buffered saline (PBS) (V group, $\mathrm{n}=16)$. Cells $(20,000$ in $0.2 \mathrm{~mL}$ PBS) were intravenously injected at 3 time-points, on day 0 , day 7 , and 2 weeks, via the tail vein in the M, N, and MSC groups. The M' group received a single injection of 20,000 Muse cells on day 0 . The $V$ group was injected with the same volume of PBS. A sham group (S group) was also prepared $(n=8)$. The dilation ratio was defined as (final diameter-initial diameter)/initial diameter. The maximum transverse diameter of the AA was measured under a microscope at both the initial operation and the final dissection.

\section{Histologic Assessment}

The aortic tissue sections were observed via Elastica-Masson staining and immunostaining, including $\alpha$ smooth muscle actin ( $\alpha \mathrm{SMA}), \mathrm{CD} 31$, and F4/ 80. Aortic medial elastin content was quantitated by the percent area of elastin compared with total medial tissue area, as described previously. ${ }^{27}$

\section{Assessment of Muse Cells Migration Dynamics}

AA-induced mice were administered 20,000 $\mathrm{GFP}^{+}$Muse cells at day 0 and euthanized at day 3 or day 5 . The whole abdominal aortas were harvested and inspected using a multiphoton laser microscope.

\section{Statistical Analysis}

Continuous variables were expressed as mean \pm standard deviation or median (interquartile range), as appropriate. Statistical analyses were performed using JMP Pro 11 software (SAS Institute, Inc, Cary, NC). The Shapiro-Wilk test for distribution normality was performed for each data set to determine the appropriate statistical testing. The ultrasound data of the aortic aneurysmal diameter were analyzed using 2-way repeated analysis of variance, followed by Bonferroni's post hoc test. The other data were analyzed with Tukey-Kramer or Steel-Dwass test, depending on the result of the test for normality.

\section{RESULTS}

\section{In Vitro Differentiation Ability of Muse Cells Into Vascular Component Cells}

The abilities of Muse cells to form clusters similar to embryonic stem cell-derived embryoid bodies in a single cellsuspension culture and to spontaneously differentiate into cells representative of 3 germ layers from those clusters, as previously reported, were reproduced (Figure 1, $A$, and Figure E2). ${ }^{19}$ These properties were not observed in nonMuse cells. ${ }^{19}$ Cells expanded from a single Muse cellderived cluster on gelatin-coated dishes contained not only $\alpha \mathrm{SMA}^{+}$cells but also $\mathrm{CD} 31^{+}$cells, suggesting that
Muse cells have the potential to generate VSMCs and ECs (Figure 1, A). On the other hand, because non-Muse cells did not generate clusters, we could not verify the spontaneous differentiation ability of non-Muse cells in vitro, as reported previously. ${ }^{19}$

The intrinsic differentiation potential of human Muse cells, endothelial progenitor cells (EPCs), and $\mathrm{CD} 34^{+}$progenitor cells into vascular component cells and the expression levels of stress-tolerant factors were compared by quantitative polymerase chain reaction. Of the EC markers, the expression of FOXC1 was highest in Muse cells compared with EPCs and CD34 $4^{+}$cells (both $P<.001$ ), whereas expression of KLF2 and MEF2C in Muse cells was moderate (Figure 1, $B$-D). Expression of ELK1, MYH10, and CAMK2 $\delta$, markers for de-differentiated VSMCs, was highest in Muse cells compared with EPCs and CD $34^{+}$cells (all at $P<.001$ ) (Figure $1, E-G$ ). Factors relevant to stress tolerance, such as HSPA8, PDIA3, and MDH1, were significantly higher in Muse cells than in EPCs and $\mathrm{CD} 34^{+}$progenitor cells (Figure $1, H-J, P<.001$ ).

To investigate the in vitro differentiation capacity of Muse cells in the AA microenvironment, co-culture of Muse cells and AA tissue was performed. Although $\mathrm{GFP}^{+}$-Muse cells were located only in the superficial layer of the aneurysmal wall at day 7, they penetrated into the deeper layer of the tissue over time at 2 weeks and 3 weeks (Figure 2, A, and Videos 1-3). When those samples were subjected to immunohistochemistry, $\mathrm{CD} 31^{+} / \mathrm{GFP}^{+}$cells and $\mathrm{CD} 34^{+} / \mathrm{GFP}^{+}$cells, markers for immature ECs, were observed in the superficial layer of the aneurysmal wall at day 7 (Figure 2, $B$ ), whereas it was not until 3 weeks that CD141/GFP ${ }^{+}$cells, a marker for mature ECs, were observed (Figure 2, $C$ ). $\alpha \mathrm{SMA}^{+} / \mathrm{GFP}^{+}$cells were observed in the tunica media at 2 weeks, and these cells later penetrated into the deeper layer of the tunica media at 3 weeks (Figure $2, C$ ). Calponin ${ }^{+} / \mathrm{GFP}^{+}$cells, a marker for differentiated VSMCs, were observed only at 3 weeks (Figure 2, C).

\section{Effect of Intravenous Injection of Human Muse Cells in Murine Aortic Aneurysm Model}

The injection protocol is shown in Figure 3, A. Representative aortic images at 8 weeks in each group are shown in Figure $3, B$. The dilation ratio remained at $1.3(0.5-2.2)$ in the $\mathrm{M}$ group at 3 weeks, whereas it was $2.4(1.8-3.0)$ in the $\mathrm{M}^{\prime}$ group, and there was no statistical difference between groups (Figure 3,C). The dilation ratio in the $\mathrm{M}$ group was statistically lower than in the $\mathrm{N}$ (median, 3.8; range, 2.3-4.2, $P<.05$ ), MSC (median, 3.4; range, 2.4$4.8, P<.05$ ), and $\mathrm{V}$ groups (median, 4.1 ; range, 3.1-5.8, $P<.05)$ at 3 weeks. Notably, the dilation ratio was not statistically different among the N, MSC, and V groups. Furthermore, although no significant difference was evident at 3 weeks between the $\mathbf{M}$ group and $\mathrm{M}^{\prime}$ group, dilation in the $\mathrm{M}$ group tended to be smaller than in the M' group. 
A
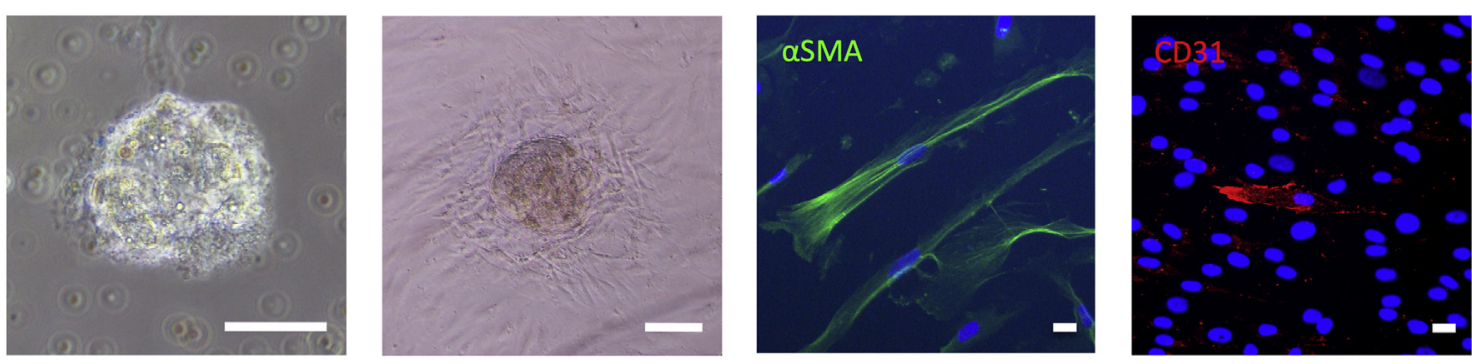

Bar $=100 \mu \mathrm{m}$

\section{Endothelial}
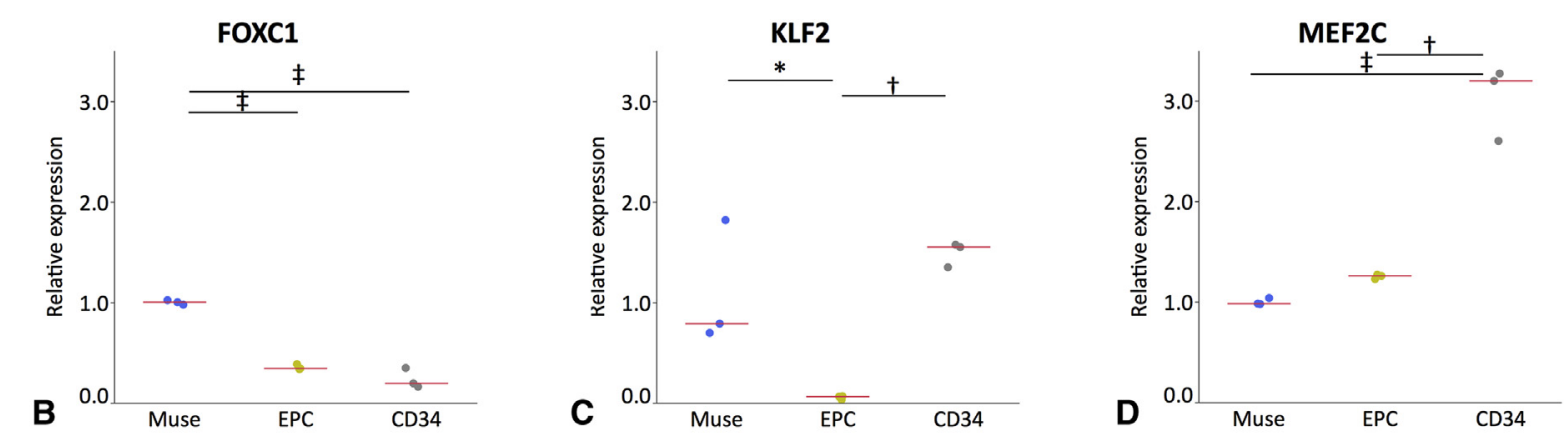

Smooth muscle
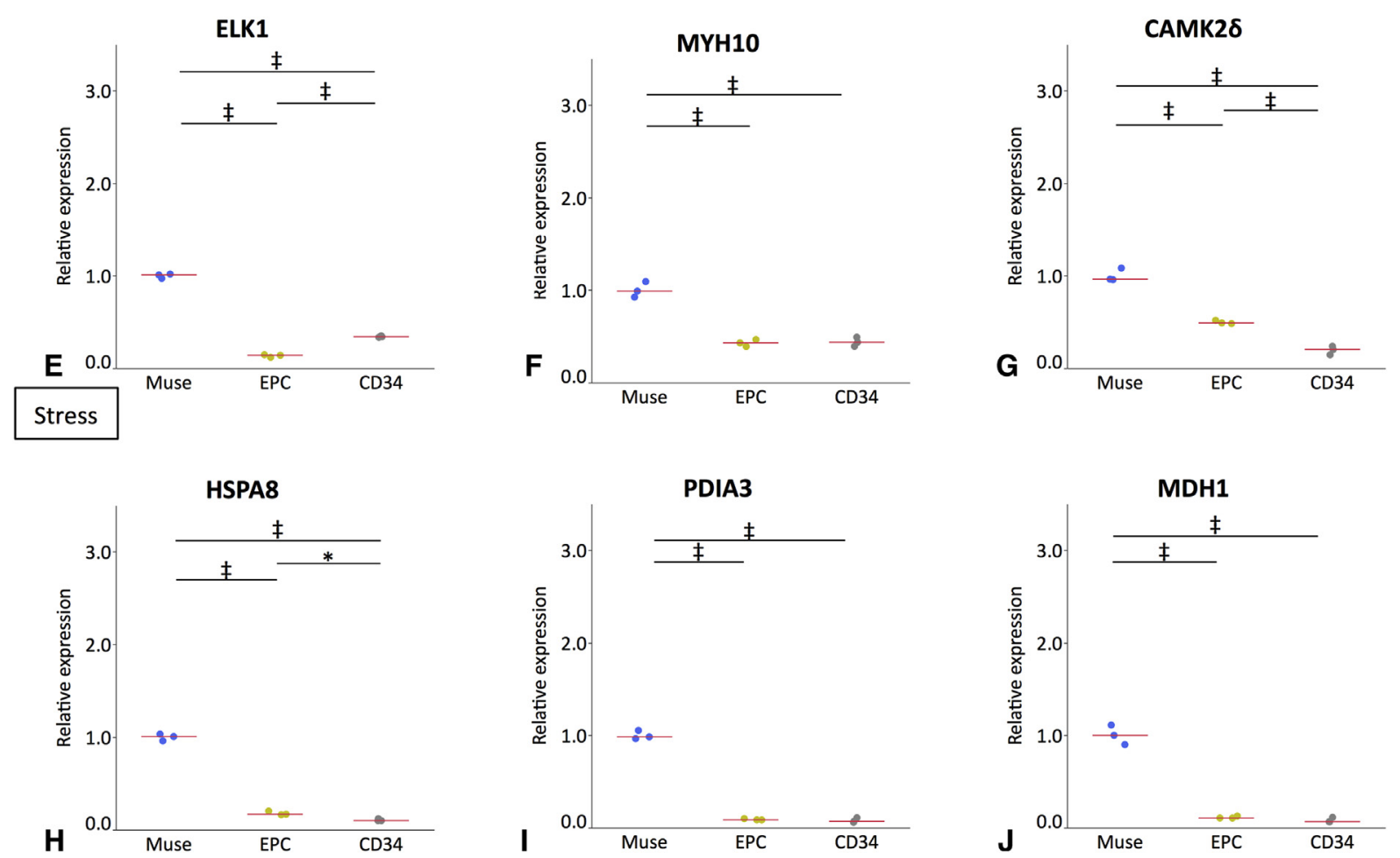

FIGURE 1. A, Representative view of a cluster formed in a single-cell suspension culture of Muse cells and adhesion culture of the cluster onto a gelatincoated dish to allow cells to expand. The expanded cells were positive for $\alpha$ SMA and CD31. B-J, Quantitative polymerase chain reaction analysis of endothelial early markers (B-D), de-differentiated smooth muscle cell markers (E-G), and stress tolerance-related markers (H-J) in Muse cells, EPCs, and $\mathrm{CD} 34+$ progenitor cells $(\mathrm{n}=3$ each). Results are shown as fold-change in gene expression normalized to $\beta$-actin. Red lines represent the median of each group. $* P<.05, \dagger P<.01, \ddagger P<.001 . \alpha S M A, \alpha$ Smooth muscle actin; Muse, multilineage-differentiating stress-enduring cell; $E P C$, endothelial progenitor cell. 


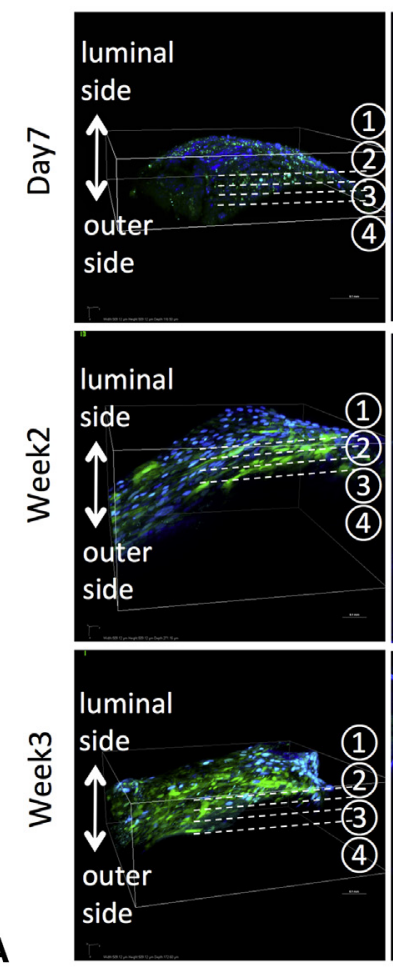

(1)

(2)

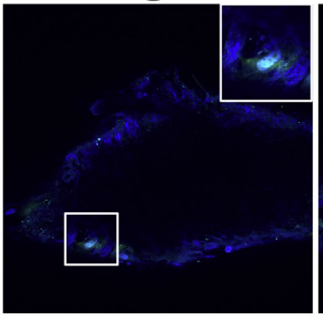

(3)

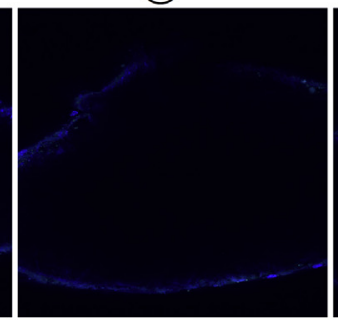

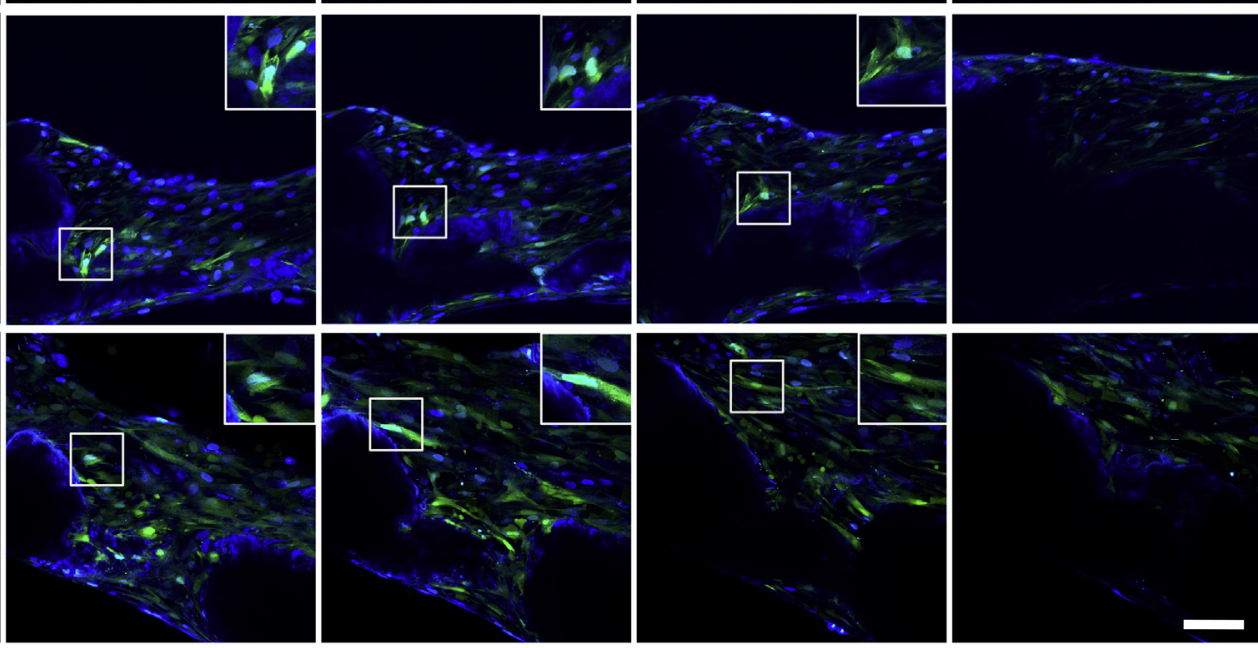

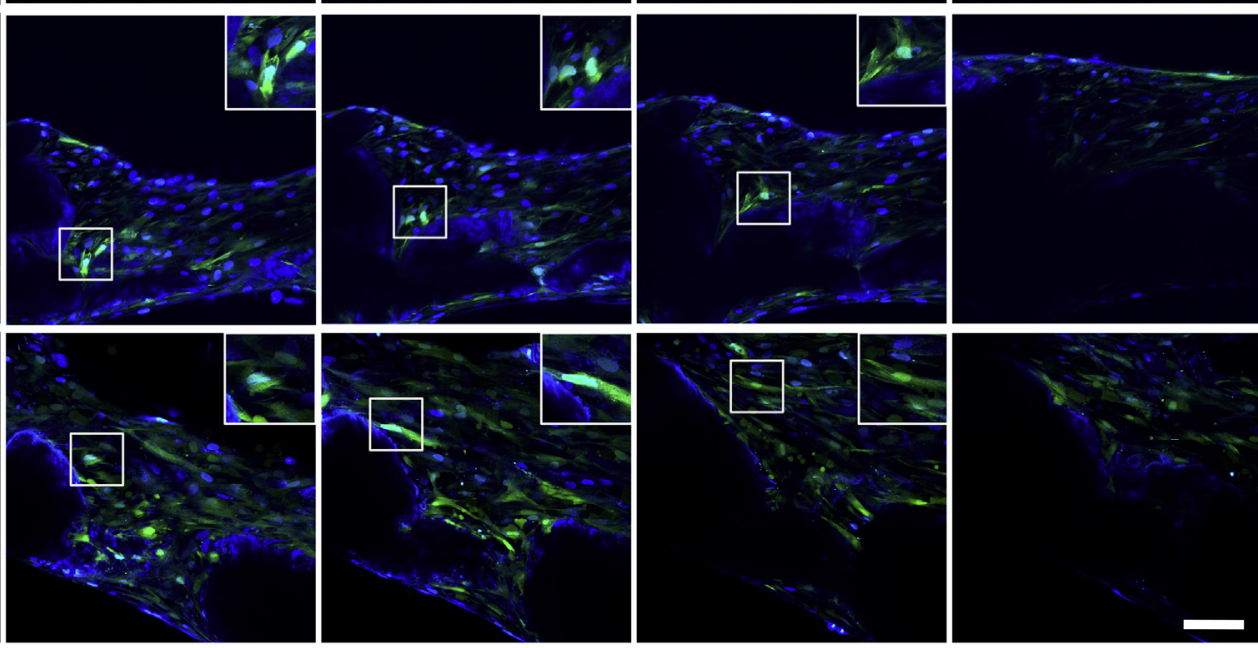

(4)

Bar $=100 \mu \mathrm{m}$
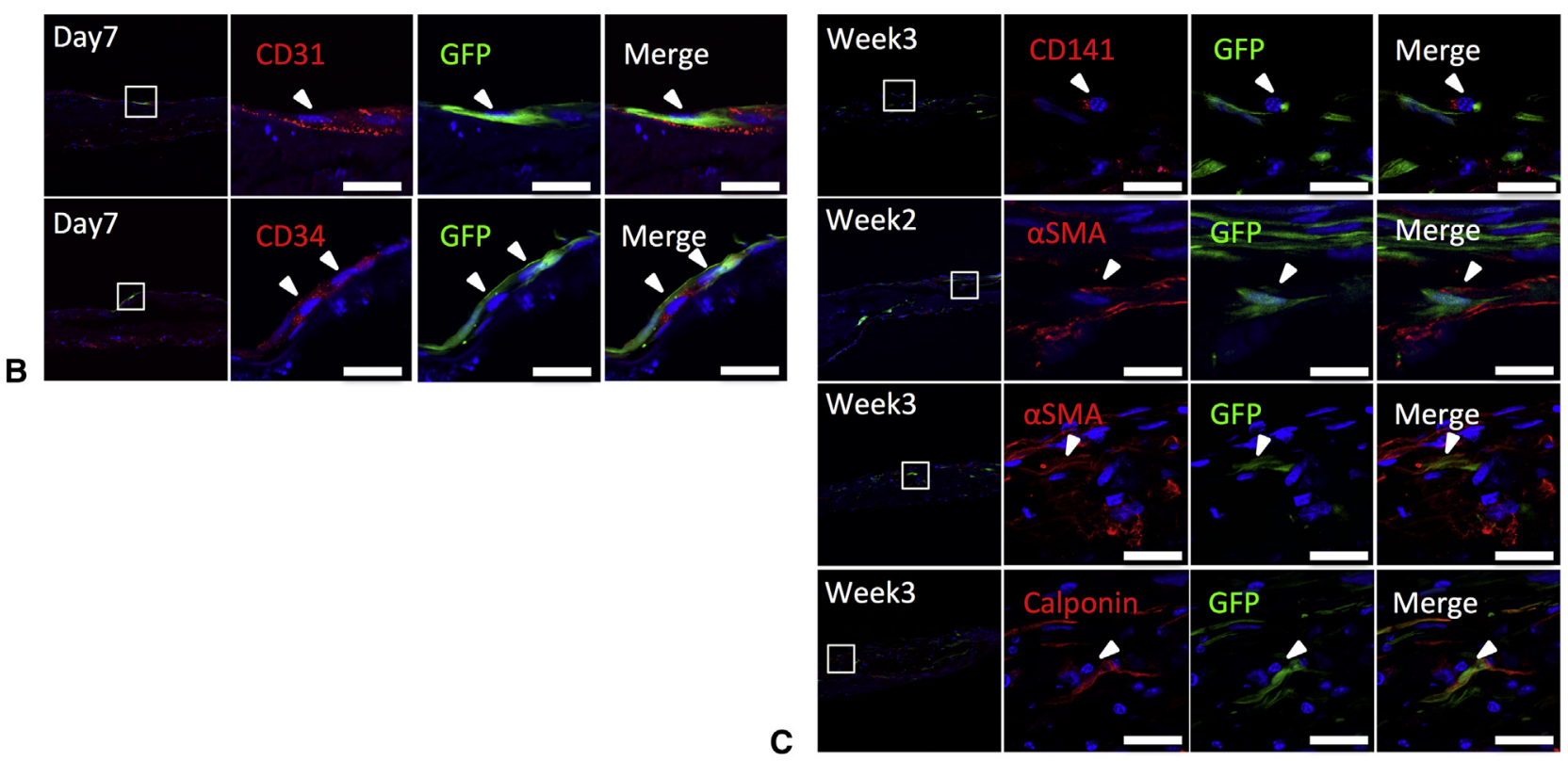

Bar $=50 \mu \mathrm{m}$

FIGURE 2. A, Representative multiphoton laser microscopy of murine AAs co-cultured with Muse cells ( $\mathrm{n}=3$ each). The left column is a 3-dimensionalreconstructed image of the aortic sample indicating the location of each axial view (dotted white lines 1-4), which was captured every $20 \mu \mathrm{m}$ from the luminal side to the outer side. B and C, Representative sagittal view of frozen cross-sections stained with CD31, CD34, CD141, $\alpha$ SMA, and calponin. Arrowheads indicate double-positive cells. GFP, Green fluorescent protein. 


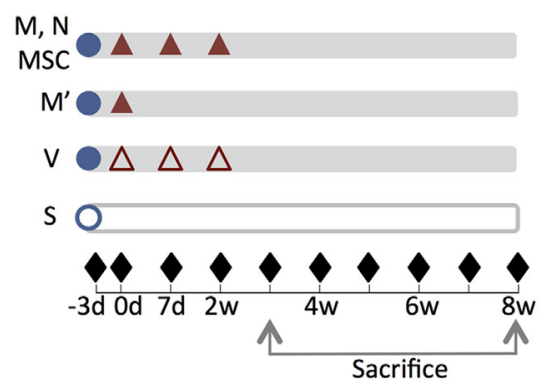

: Aneurysm induction $\triangle:$ : PBS infusion : Sham operation A $\mathbf{\Lambda}:$ Cell infusion

Week 3

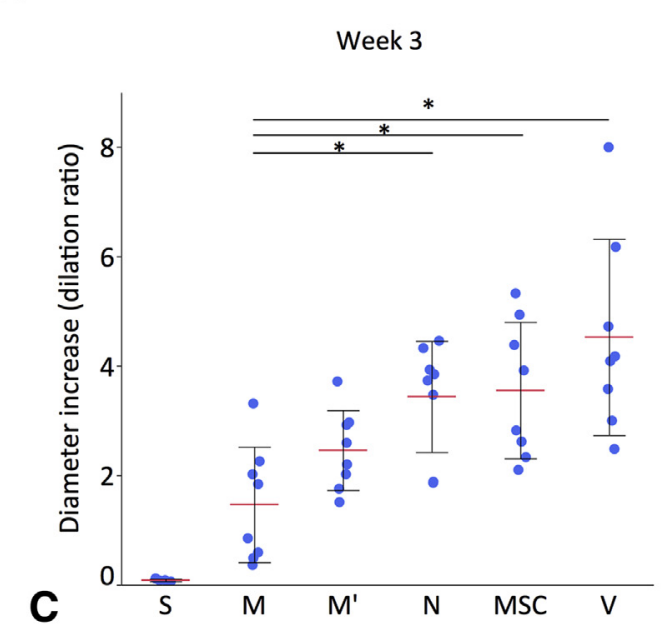

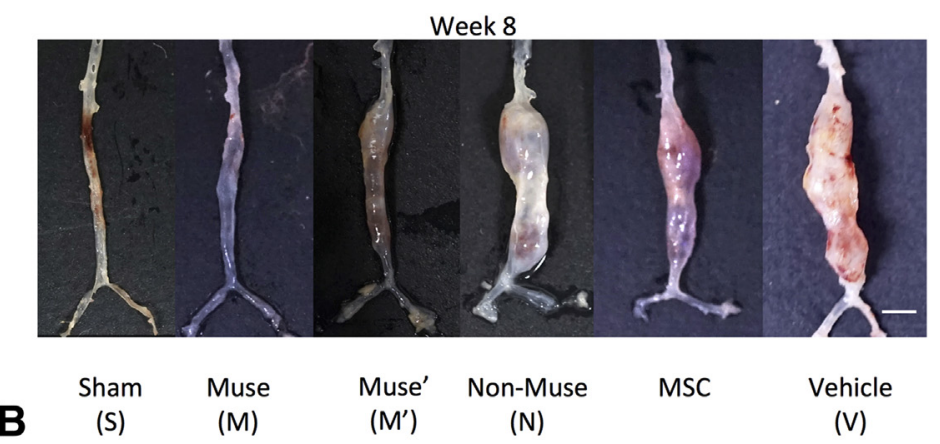

Bar $=2 \mathrm{~mm}$
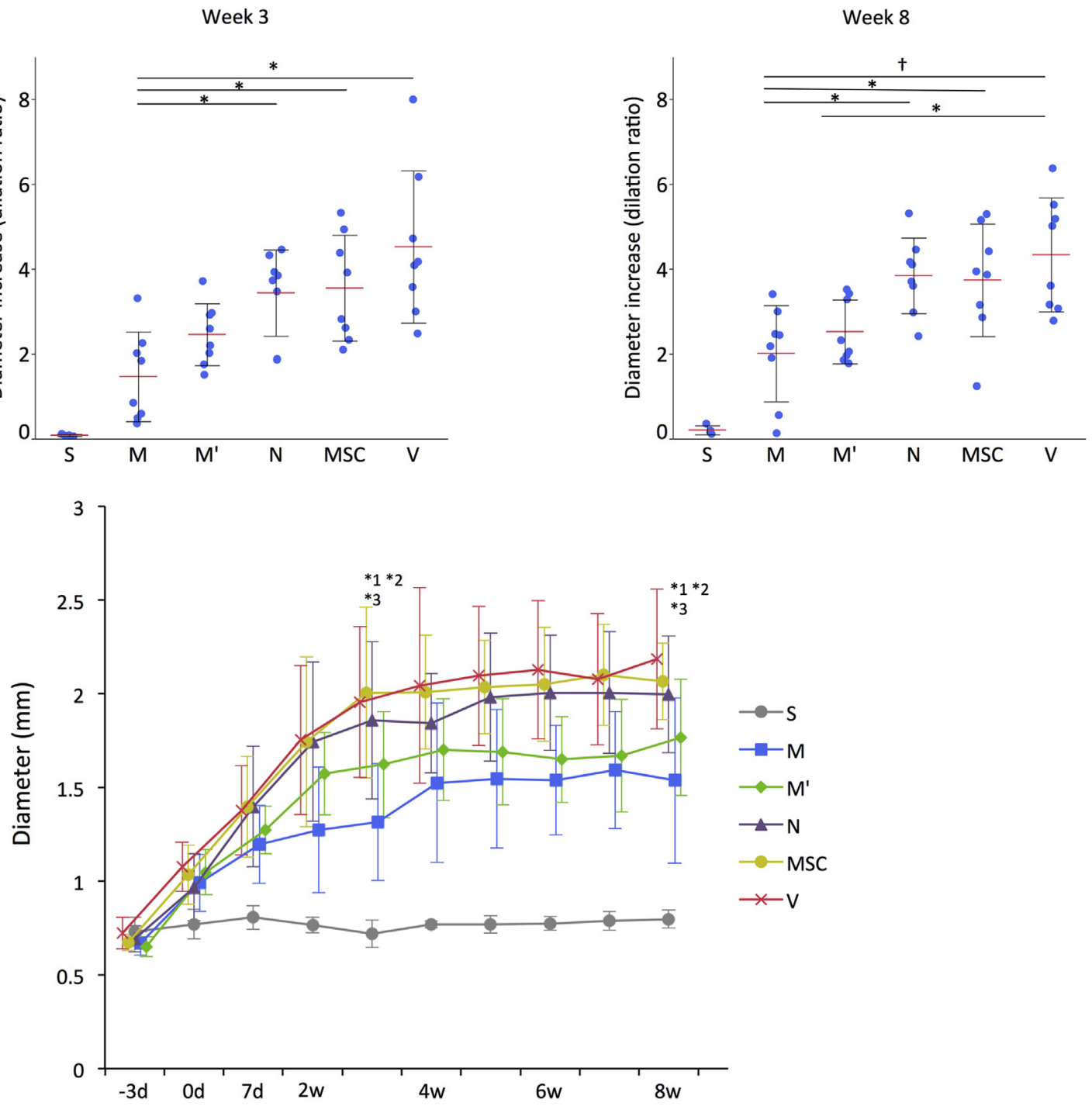

D

*1: M vs N, *2: M vs MSC, *3: M vs V

FIGURE 3. A, Schematic representation of our in vivo study protocol. B, Macroscopic view of the AA at 8 weeks. C, Dilation ratio of the AA. Red lines represent the mean of each group. D, Diameter measurement of the AA by ultrasound inspection. S, sham group ( $\mathrm{n}=8$ ); $M$, Muse group (multiple injection) $(\mathrm{n}=16) ; M^{\prime}$, Muse group (single injection) $(\mathrm{n}=16) ; N$, non-Muse group $(\mathrm{n}=16)$; MSC, MSC group $(\mathrm{n}=16) ;$, vehicle group ( $\left.\mathrm{n}=16\right)$. Error bars represent the standard deviation. $* P<.05, \dagger P<.01$. $P B S$, Phosphate-buffered saline; Muse, multilineage-differentiating stress-enduring cell; $M S C$, mesenchymal stem cell. 


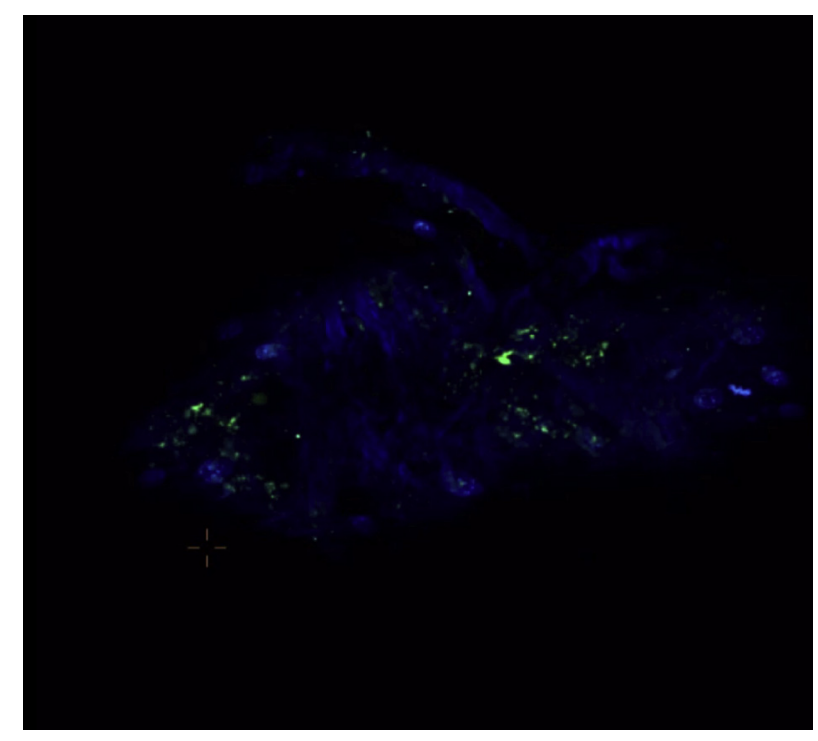

VIDEO 1. Multiphoton laser microscopic movie of murine AAs cocultured with $\mathrm{GFP}^{+}$-Muse cells for 1 week. The movie is shown from the luminal side to the outer side. Video available at: http://www.jtcvsonline. org/article/S0022-5223(18)30409-4/fulltext.

The same tendency was observed at 8 weeks (Figure 3, $C)$. The dilation ratio was $1.9 \pm 1.1$ in the $\mathrm{M}$ group and $2.8 \pm 0.6$ in M' group, and the difference was not significant. The dilation ratio in both groups was significantly lower than in the $\mathrm{V}$ group, which was $4.3 \pm 1.3(P<.01$ and $P<.05$, respectively). In addition, the dilation ratio in the $\mathrm{M}$ group was substantially smaller than in the $\mathrm{N}$ $(3.8 \pm 0.8, P<.05)$ or MSC groups $(3.7 \pm 0.7, P<.05)$. Dilation in the N, MSC, and V groups was similar, and there was no significant difference among them.

Figure $3, D$, shows the aneurysmal diameter change over time in each group measured by ultrasound. In the M and M'

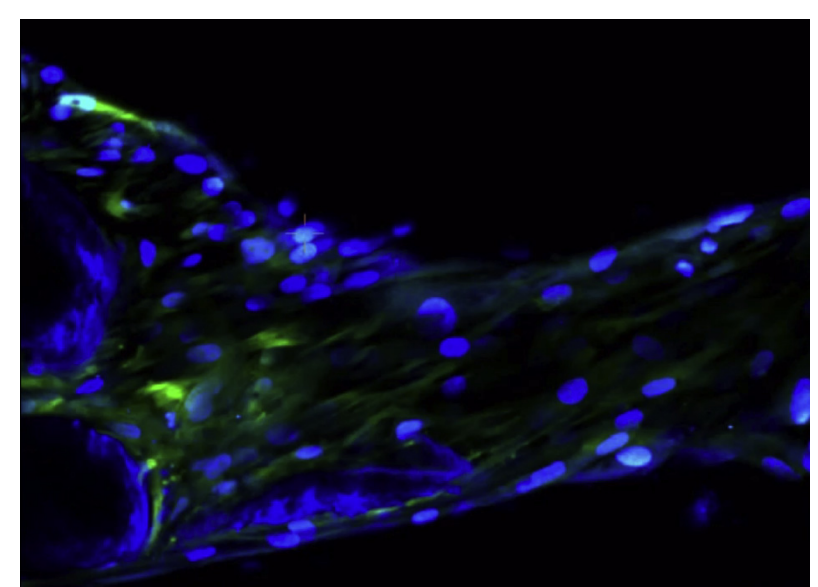

VIDEO 2. Multiphoton laser microscopic movie of murine AAs co-cultured with $\mathrm{GFP}^{+}$-Muse cells for 2 weeks. The movie is shown from the luminal side to the outer side. Video available at: http://www. jtcvsonline.org/article/S0022-5223(18)30409-4/fulltext.

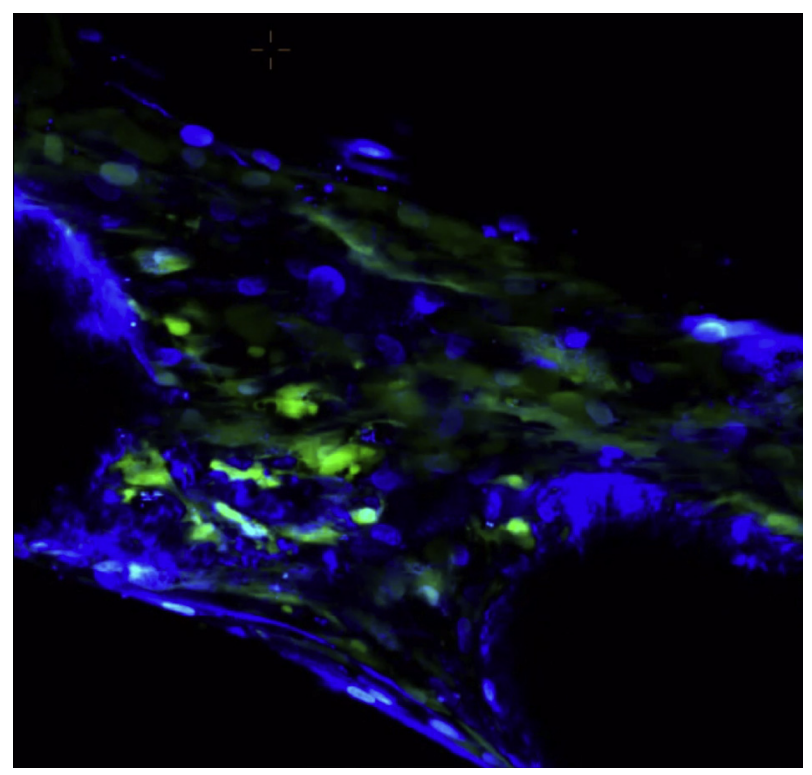

VIDEO 3. Multiphoton laser microscopic movie of murine AAs co-cultured with $\mathrm{GFP}^{+}$-Muse cells for 3 weeks. The movie is shown from the luminal side to the outer side. Video available at: http://www.jtcvsonline.org/ article/S0022-5223(18)30409-4/fulltext.

groups, the diameter was minimal after day 7, and the diameters of both groups were significantly smaller than those in the N, MSC, and V groups at 3 weeks and 8 weeks. The change in aneurysm diameter was not significantly different between the M group and M' group for the entire period.

\section{Histologic Assessment}

Figure 4 shows Elastica-Masson staining of the aortic tissue sections at 3 weeks. In the $M$ group, the ratio of the aortic medial elastin content was $13.7 \% \pm 4.4 \%$, significantly higher than that of the $\mathrm{N}(6.4 \% \pm 1.5 \%)$, MSC $(4.8 \% \pm 4.2 \%)$, and $\mathrm{V}$ groups $(5.2 \% \pm 2.1 \%$; all at $P<.01)$, whereas there was no significant difference between the $\mathbf{M}$ and $M$ ' groups (Figure $4, B$ ). The ratio was $10.5 \% \pm 3.0 \%$ in the $M^{\prime}$ group and was significantly higher than in the MSC and V groups. The ratio was not significantly different among the N, MSC, and V groups.

Integration of Muse cells in the AA was assessed by immunostaining (Figure 5). In the $\mathrm{M}$ group, $\alpha \mathrm{SMA}^{+} / \mathrm{GFP}^{+}$cells were detected among $\mathrm{GFP}^{+}$cells at a frequency of $71.9 \pm$ 27.0 cells $/ \mathrm{mm}^{2}$ at 3 weeks and $57.3 \pm 36.3$ cells $/ \mathrm{mm}^{2}$ at 8 weeks (Figure 5, $A$ ). In contrast, the number of $\alpha \mathrm{SMA}^{+}$/ $\mathrm{GFP}^{+}$cells in the $\mathrm{M}^{\prime}, \mathrm{N}$, and MSC groups was significantly smaller than in the $\mathrm{M}$ group (all at $P<.01$ ) and similar to that in the $\mathrm{M}^{\prime}$ group $(P<.01$ at 3 weeks, $P<.05$ at 8 weeks $)$. No $\mathrm{GFP}^{+}$cells were detected in the thoracic aorta in all these groups (data not shown).

In the $\mathrm{M}$ group, $\mathrm{CD} 31^{+} / \mathrm{GFP}^{+}$cells were detected at a frequency of $25.8 \pm 14.5$ cells $/ \mathrm{mm}^{2}$ at 3 weeks and $22.5 \pm 11.2$ cells $/ \mathrm{mm}^{2}$ at 8 weeks (Figure $5, B$ ). In contrast, only a small number of double-positive cells was counted 
Hosoyama et al

Week 3
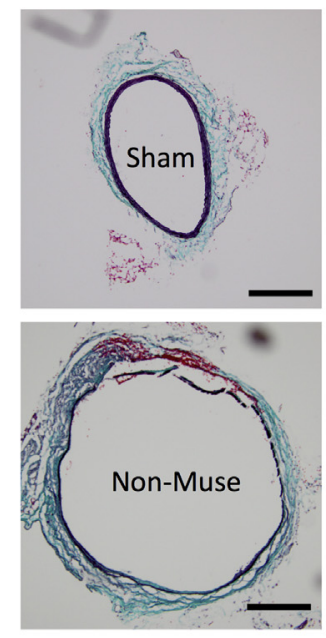

A
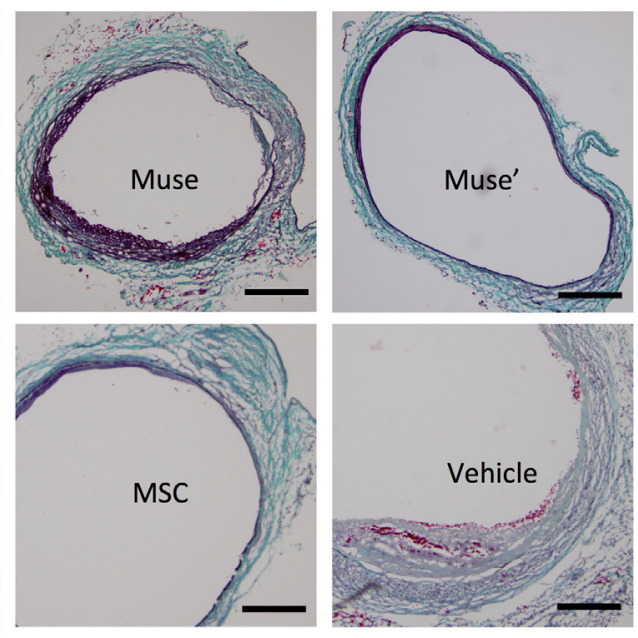

Bar $=300 \mu \mathrm{m}$

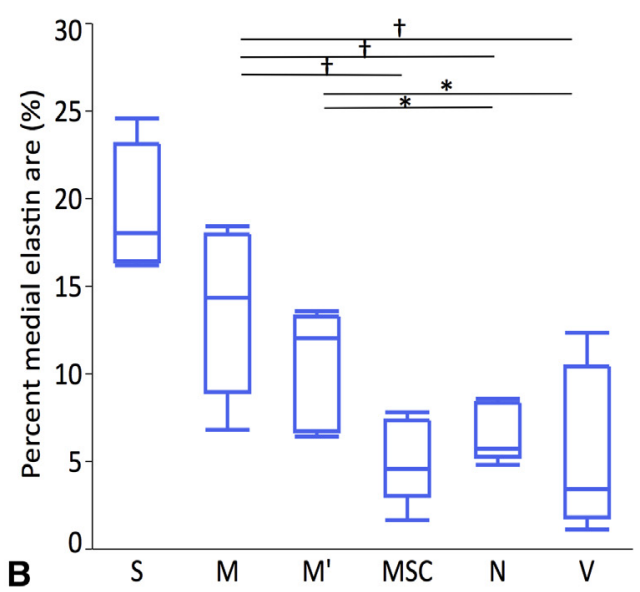

FIGURE 4. A, Elastica-Masson staining of AAs at 3 weeks. B, Quantitative analysis of the medial elastin area. $S$, sham group ( $\mathrm{n}=4$ ); $M$, Muse group (multiple injection) $(\mathrm{n}=8) ; M^{\prime}$, Muse group (single injection) $(\mathrm{n}=8) ; N$, non-Muse group $(\mathrm{n}=8) ;$ MSC, MSC group $(\mathrm{n}=8) ; V$, vehicle group $(\mathrm{n}=8)$. The upper and lower borders of the box represent the upper and lower quartiles. The middle horizontal line represents the median. The upper and lower whiskers represent the maximum and minimum values of nonoutliers. ${ }^{*} P<.05, \dagger P<.01$. MSC, Mesenchymal stem cell; Muse, multilineage-differentiating stress-enduring cell.

in the $\mathrm{N}$ and MSC groups (all at $P<.01$ ). In the $\mathrm{M}^{\prime}$ group, the number of double-positive cells was $19.8 \pm 13.2 \mathrm{cells} / \mathrm{mm}^{2}$ at 3 weeks, similar to that in the $\mathrm{M}$ group, whereas the number in the M' group decreased to $6.7 \pm 6.7$ cells $/ \mathrm{mm}^{2}$ at 8 weeks, which was significantly lower than in the $\mathrm{M}$ group $(P<.01)$.

Anti-inflammatory effects were also assessed (Figure 5, $C$ ). In the $\mathrm{M}, \mathrm{M}^{\prime}, \mathrm{N}$, and MSC groups, the number of $\mathrm{F} 4 / 80^{+}$ cells was significantly smaller than in the $\mathrm{V}$ group, although the differences were not statistically significant among these 4 groups. Nevertheless, the number of $\mathrm{F} 4 / 80^{+}$cells was lowest in the $\mathrm{M}$ group $\left(3.3 \pm 1.2\right.$ cells $\left./ 0.01 \mathrm{~mm}^{2}\right)$ at 3 weeks and 8 weeks $\left(3.3 \pm 1.1\right.$ cells $\left./ 0.01 \mathrm{~mm}^{2}\right)$.

\section{Tissue Distribution of Muse Cells}

We next addressed the tissue distribution of intravenously injected Muse and non-Muse cells by determining the expression level of the human specific Alu sequence in the abdominal aorta, lung, spleen, liver, heart, and kidney using quantitative polymerase chain reaction at 8 weeks. Expression of human Alu in the abdominal aorta in the M group was 13.2-fold higher than in the $\mathrm{N}$ group (Figure 6). Human Alu was not detected in the other organs in the M group, except for low levels of expression in the lung and spleen compared with that in the aorta. In the $\mathrm{N}$ group, a very low level of human Alu was detected only in the abdominal aorta and not in other organs.

\section{Muse Cells Homed Into the Aortic Aneurysm From the Adventitial Side}

To examine how Muse cells migrate to and integrate into aneurysmal tissue, we inspected the post-transplanted murine aortas in the early phase by multiphoton laser microscopy (Figure 7, A, and Videos 4 and 5). In contrast to the in vitro co-culture experiments shown in Figure 2, A, $\mathrm{GFP}^{+}$Muse cells were detected only at the outermost layer of the vasculature on day 3 , and, in some parts, $\mathrm{GFP}^{+}$Muse cells accumulated around a vasa vasorum-like vasculature structure in the tunica externa (Figure 7, A). On day 5, $\mathrm{GFP}^{+}$Muse cells still remained at the tunica externa, but expanded toward the tunica media and luminal layers of the aortic wall.

\section{DISCUSSION}

This study is the first to demonstrate the therapeutic efficacy of human Muse cells for aneurysmal degeneration in an animal model. The novel findings are (1) Muse cells have the potential to differentiate into vascular component cells, both into VSMCs and ECs; (2) after intravenous injection, Muse cells homed into the AA from the adventitial side and expanded to the aortic tissue; (3) after integration, Muse cells differentiated spontaneously into VSMCs and ECs. Concomitantly, elastic fibers were preserved and macrophage invasion was efficiently suppressed. These Muse cell features together led to substantial attenuation of AA dilation.

\section{Muse Cells Differentiate Into Vascular Lineage Cells}

We demonstrated that Muse cells have the ability to spontaneously differentiate into vascular component cells in an in vitro system and in a co-culture experiment. These findings revealed that the differentiation potential of Muse cells spans the vascular component cells, 

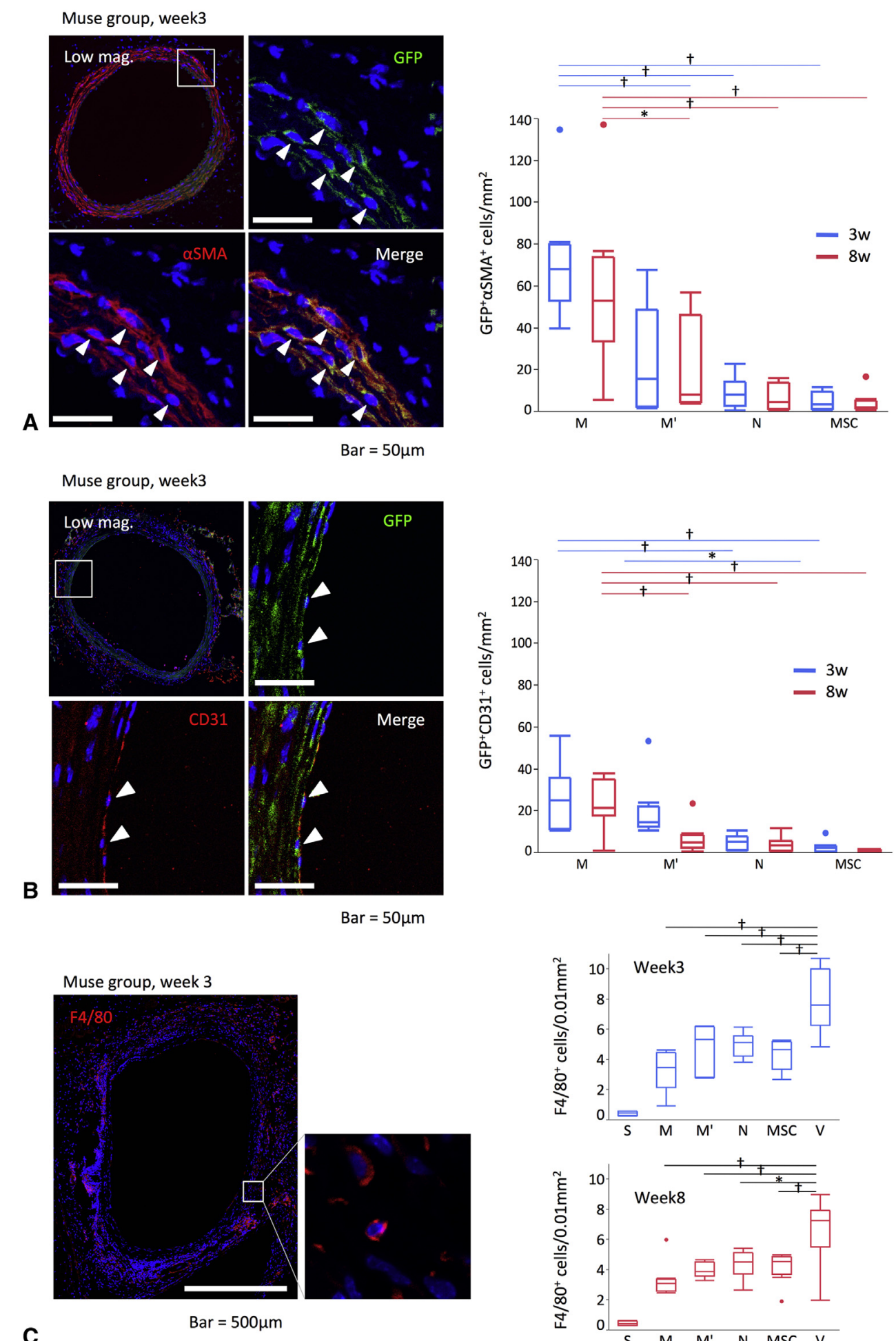

$\mathrm{Bar}=50 \mu \mathrm{m}$
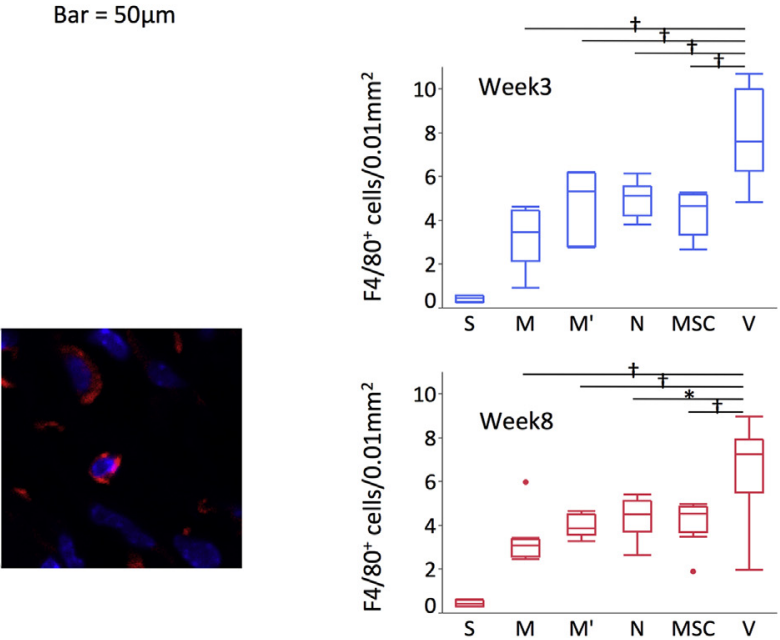

FIGURE 5. A, $\alpha$ SMA and GFP immunostaining of the AAs at 3 weeks, along with quantitative analysis. B, CD31 and GFP immunostaining and quantitative analysis of AAs at 3 weeks. C, F4/80 immunostaining and quantitative analysis of AAs at 3 weeks. Arrowheads indicate double-positive cells. $S$, sham group ( $\mathrm{n}=8) ; M$, Muse group (multiple injection) $(\mathrm{n}=16) ; M^{\prime}$, Muse group (single injection) $(\mathrm{n}=16) ; N$, non-Muse group ( $=16$ ); MSC, MSC group $(\mathrm{n}=16) ; \mathrm{V}$, vehicle group $(\mathrm{n}=16)$. The upper and lower borders of the box represent the upper and lower quartiles. The middle horizontal line represents the median. The upper and lower whiskers represent the maximum and minimum values of nonoutliers. Extra dots represent outliers. $* P<.05$, $\dagger P<.01 . \alpha S M A, \alpha$ Smooth muscle actin; GFP, green fluorescence protein; MSC, mesenchymal stem cell; Muse, multilineage-differentiating stress-enduring cell. 


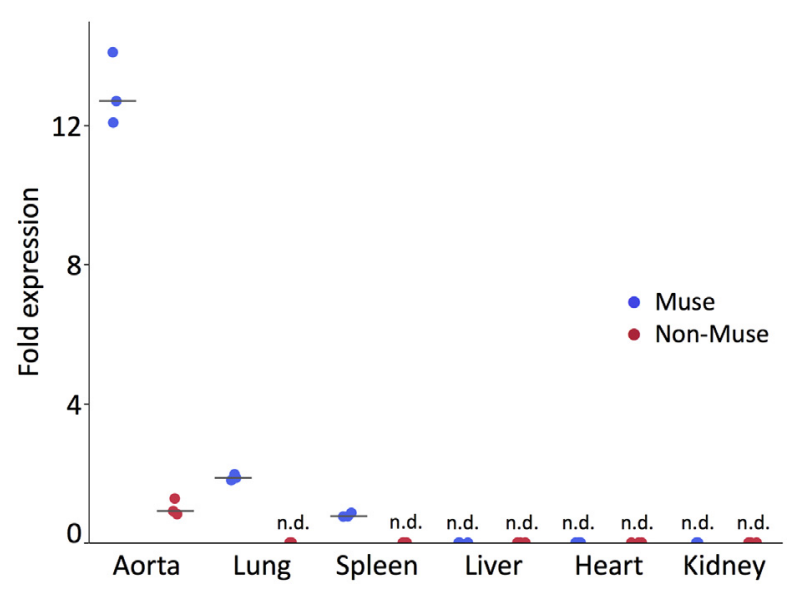

FIGURE 6. Quantitative polymerase chain reaction analysis of human Alu expression ( $n=3$ each). Results are shown as fold-change in gene expression. Black lines represent the median of each group. Muse, Multilineage-differentiating stress-enduring cell; $n . d$., not detected.

VSMCs, and ECs. CD34 ${ }^{+}$progenitor cells contain vascular progenitor cells, and although they do not spontaneously differentiate in vivo, they can differentiate into both ECs and VSMCs under a specific cytokine induction system. ${ }^{28}$ Notably, expression of VSMC de-differentiation-related markers was generally higher in Muse cells than EPCs and CD34 ${ }^{+}$progenitor cells. Because VSMCs are a pivotal element that regulates structural strength of aortic tissue, the high ability of Muse cells to differentiate into VSMCs is one of the advantages of Muse cells for treating AAs.

\section{Muse Cell Administration and Distinct Migration}

Many previously reported cell therapies for AA are based on invasive localized administration of cells, which often requires time for preparation of a huge number of cells, that is, at least 1 million cells, because the cells basically do not selectively integrate into AA or remain in the tissue, so that a large number of administered cells is considered necessary to deliver the desired effects. ${ }^{13-15}$ In contrast, the number of Muse cells supplied in the present study was only 60,000 , that is, 20,000 cells 3 times, by intravenous injection, a simple and highly noninvasive method. High expression of stress-tolerant factors in Muse cells might be related to the high proportion of cell survival and integration into AA tissue. Stress tolerance allowed these cells to survive in the AA tissue where proapoptotic and proinflammatory factors are abundant. $^{29}$ On the other hand, non-Muse cells and MSCs exhibited modest effects possibly attributed to trophic factors. This similarity is convincing because approximately $96 \%$ of MSCs are composed of nonMuse cells, and the trophic effect is considered to be similar. Minimization of cell number is important for practical concerns because it will decrease both the time and the cost required to prepare cells and decrease the risks of adverse effects, such as embolic events. Furthermore, allogeneic Muse cells would be more feasible for clinical application than autologous cells. Previously, Muse cells were demonstrated to have immunodulatory effects. $^{22,30}$ If allogeneic Muse cells are effective for treating AA, they could be an "off-theshelf" cell source for AA therapy.

The mode of action of Muse cells was also unique: In an in vivo system, the Muse cells migrated into the AA tissue through the vasa vasorum-like vasculature structure, homed into the tunica externa first, and then migrated to the luminal side through tunica media. So far, there are no reports demonstrating the route of cell migration related to cell therapies for AA, and in that sense, Muse cell homing into the AA from the adventitial side is a novel finding. Several factors have been reported to control migration of MSCs, such as stromal cell-derived factor-1 (SDF-1)-C$\mathrm{X}-\mathrm{C}$ chemokine receptor type 4 (CXCR4) axis. ${ }^{31}$ Because Muse cells are a subpopulation of MSCs, Iseki and colleagues $^{32}$ reported the involvement of CXCR4 regarding Muse cell migration. In their in vitro migration assay, migration of Muse cells was partially suppressed by AMD3100, a CXCR4 antagonist, whereas migration of non-Muse cells was completely abrogated. The authors concluded that CXCR4 is suggested to be one of the key receptors to mediate Muse cell migration, although other critical factors also appear to be responsible.

\section{Muse Cells Attenuate Aortic Aneurysm}

In previously reported cell therapies, ${ }^{7-9,11,17,18}$ trophic effects and matrix metalloproteinase inhibition were major actions. In contrast, our present study illustrated that Muse cell efficiently differentiated into VSMCs and ECs. The elastic fibers in the murine AA tissue treated with Muse cells were also substantially preserved. These findings indicated that the attenuation of the AAs evolved from VSMCs and ECs that spontaneously differentiated from migrated Muse cells, and possibly subsequent preservation of ECM, including elastic fibers. In this study, we also elucidated the damaged tissue-limited homing of Muse cells. The human Alu sequence localization analysis showed that infused Muse cells mostly accumulated in the aorta, and not in other organs, such as the liver and kidney; they were accumulated at a very low level in the lung and spleen at 8 weeks, consistent with the previous report demonstrating that administrated Muse cells was detected only in chemically injured liver, not even in BM where Muse cells reside normally. ${ }^{32}$ This characteristic may be due to the significant attenuation of Muse cell treatment at 8 weeks, whereas Yamawaki-Ogata and colleagues ${ }^{14}$ reported that the efficacy of MSCs disappeared within 8 weeks. 
(1)
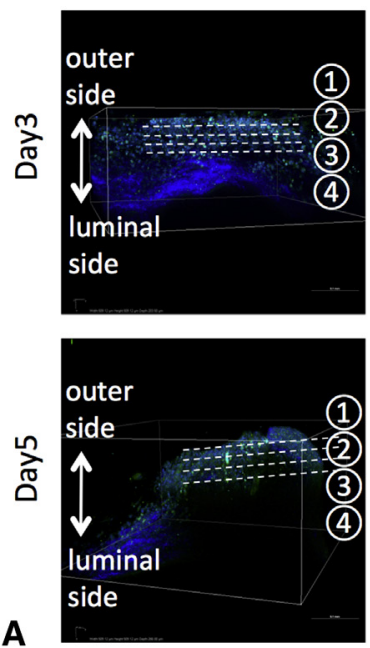

(2)
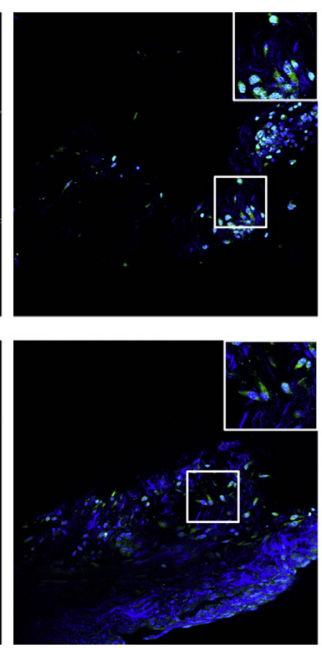

(3)
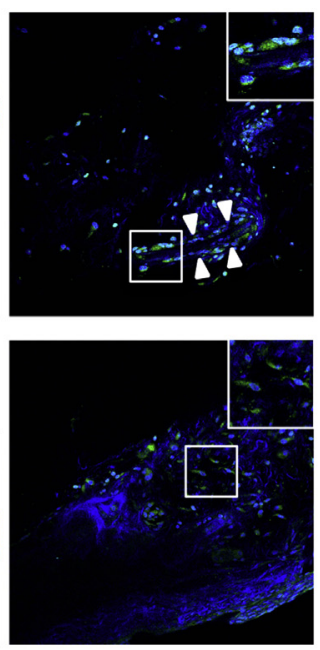
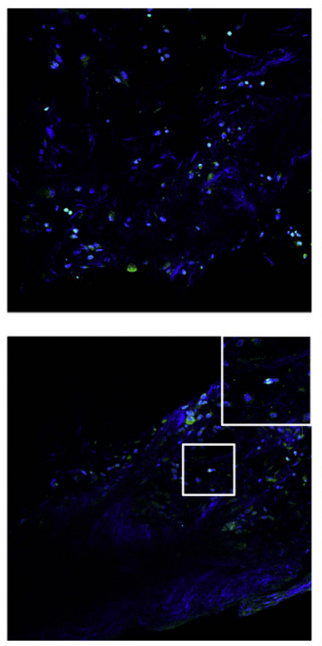

(4)
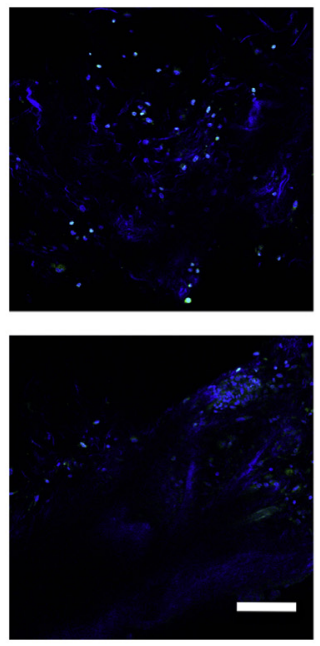

$\operatorname{Bar}=100 \mu \mathrm{m}$

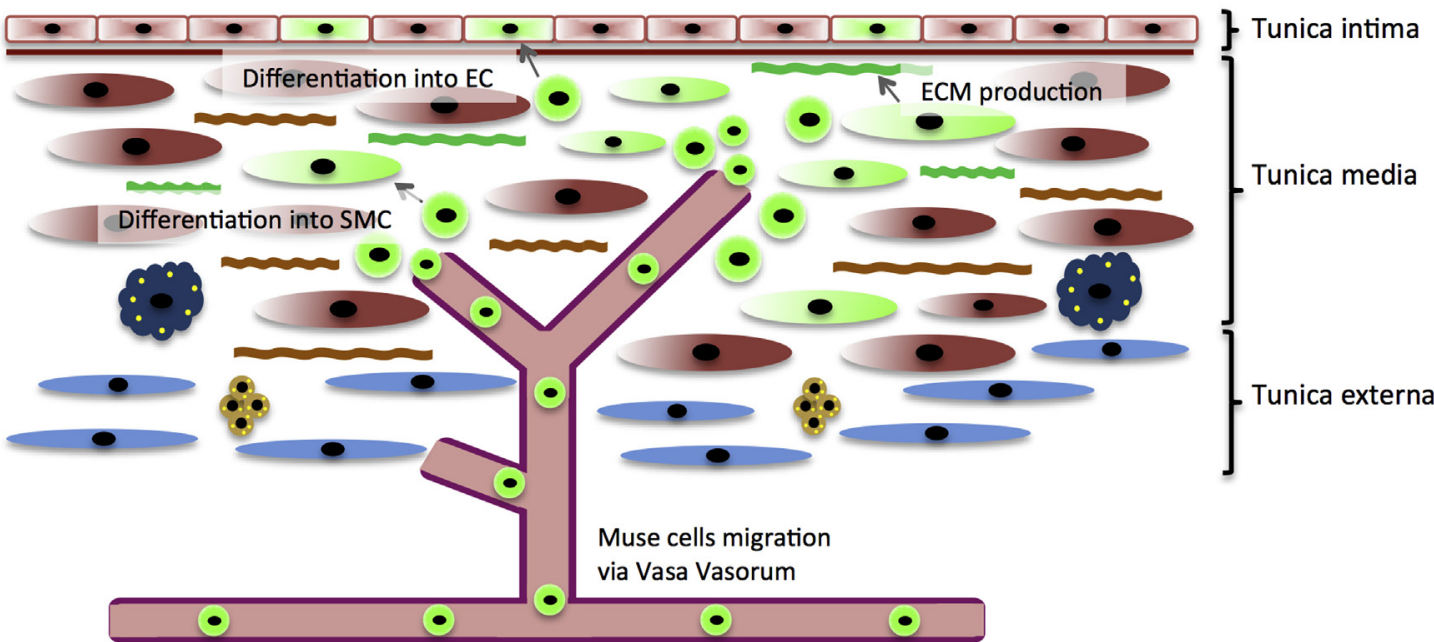

B

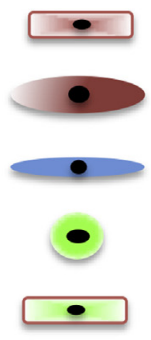

Endothelial cell

Muse-derived smooth muscle cell

Smooth muscle cell

Fibroblast

Muse cell

Muse-derived Endothelial cell

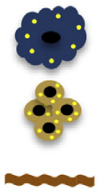

Macrophage

T-lymphocyte

ECM

FIGURE 7. A, Representative multiphoton laser microscopy image of AAs harvested at day 3 and day 5 ( $\mathrm{n}=3$ each). The left column is a 3 -dimensionalreconstructed image of the aortic sample indicating the location of each axial view (dotted white lines 1-4), captured every $20 \mu \mathrm{m}$ from the outer side to the luminal side. Arrowheads indicate vasa vasorum-like vasculature structure in the tunica externa. B, Hypothetic model of Muse cells treating AAs. EC, Endothelial cell; ECM, extracellular matrix; SMC, smooth muscle cell; Muse, multilineage-differentiating stress-enduring cell. 


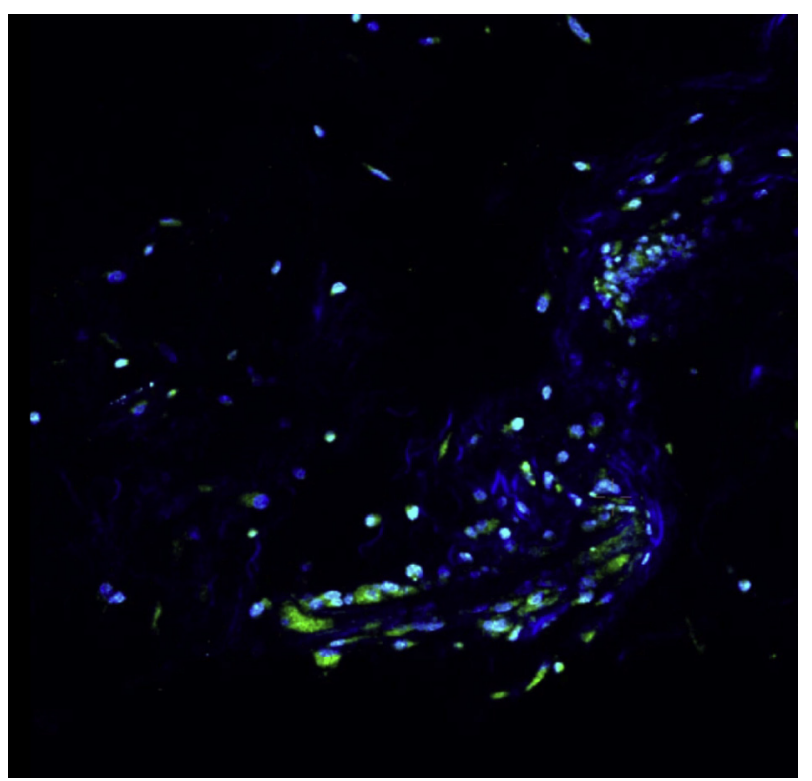

VIDEO 4. Multiphoton laser microscopic movie of murine AAs harvested at day 3. Muse cells were labeled with GFP before administration. The movie is shown from the outer side to the luminal side. Video available at: http://www.jtcvsonline.org/article/S0022-5223(18)30409-4/fulltext.

\section{Study Limitations and Future Directions}

In the present study, we performed the cell infusion in the acute phase of AA, day 3, as an early-stage intervention for AA. Further studies are required to elucidate the efficacy of Muse cells for chronic aneurysms. Investigation of other types of animal AA models, particularly the angiotensin-

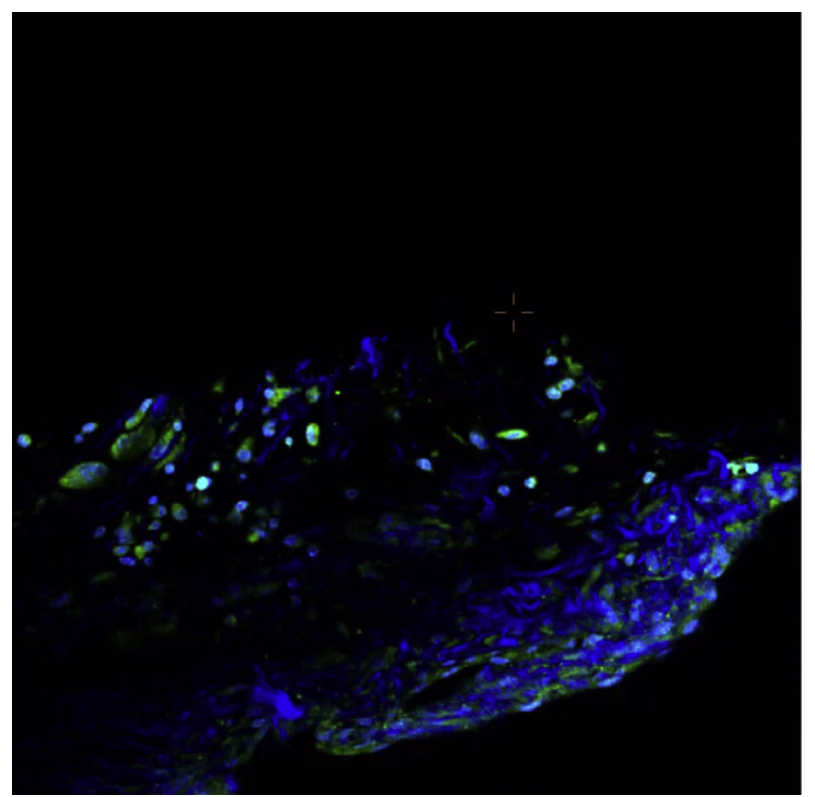

VIDEO 5. Multiphoton laser microscopic movie of murine AAs harvested at day 5. Muse cells were labeled with GFP before administration. The movie is shown from the outer side to the luminal side. Video available at: http:// www.jtcvsonline.org/article/S0022-5223(18)30409-4/fulltext.
II-infused apolipoprotein E-knockout murine model, is warranted to clarify the broad applicability of Muse cells. Because the AA was induced in murine abdominal aorta, these results may not be applicable to the thoracic AA. Using SCID mice is a possible factor affecting administered cell engraftment. Allogenic and autologous transplant of Muse cells need to be examined in future studies. The paracrine effects of Muse cells need to be evaluated in further studies, which has been regarded as a main mechanism of MSCs. In this study, we did not inspect the reaction of endogenous Muse cell against AA induction in the murine model. Because Muse cells reportedly comprise approximately $0.03 \%$ of the mononucleated fraction in the $\mathrm{BM},{ }^{19}$ the therapeutic effects are presumed to be limited. However, at least some of the endogenous Muse cells are supposed to migrate into AA tissue. Further studies are needed. Considering the multiple variables tested, there is a risk of a type 1 error.

\section{CONCLUSIONS}

After intravenous injection, Muse cells homed and expanded to the aneurysm from the adventitial side. Subsequently, Muse cells differentiated spontaneously into VSMCs and ECs, and elastic fibers were preserved. These Muse cell features together led to substantial attenuation of aneurysmal dilation.

\section{Conflict of Interest Statement}

K.H., S.W., Y.K., F.O., and M.D. are in a co-development agreement with Life Science Institute, Inc (LSII; Tokyo, Japan) for the use of Muse cells. All other authors have nothing to disclose with regard to commercial support.

\section{References}

1. Traub O, Berk BC. Laminar shear stress: mechanisms by which endothelial cells transduce an atheroprotective force. Arterioscler Thromb Vasc Biol. 1998;18: 677-85.

2. Visse R, Nagase H. Matrix metalloproteinases and tissue inhibitors of metalloproteinases: structure, function, and biochemistry. Circ Res. 2003;92:827-39.

3. Kazi M, Thyberg J, Religa P, Roy J, Eriksson P, Hedin U, et al. Influence of intraluminal thrombus on structural and cellular composition of abdominal aortic aneurysm wall. J Vasc Surg. 2003;38:1283-92.

4. Fontaine V, Touat Z, Mtairag el M, Vranckx R, Louedec L, Houard X, et al. Role of leukocyte elastase in preventing cellular re-colonization of the mural thrombus. Am J Pathol. 2004;164:2077-87.

5. Sakalihasan N, Limet R, Defawe OD. Abdominal aortic aneurysm. Lancet. 2005; 365:1577-89.

6. Motoki T, Kurobe H, Hirata Y, Nakayama T, Kinoshita H, Rocco KA, et al. PPAR-gamma agonist attenuates inflammation in aortic aneurysm patients. Gen Thorac Cardiovasc Surg. 2015;63:565-71.

7. Allaire E, Muscatelli-Groux B, Guinault AM, Pages C, Goussard A, Mandet C, et al. Vascular smooth muscle cell endovascular therapy stabilizes already developed aneurysms in a model of aortic injury elicited by inflammation and proteolysis. Ann Surg. 2004;239:417-27.

8. Allaire E, Muscatelli-Groux B, Mandet C, Guinault AM, Bruneval P, Desgranges $\mathrm{P}$, et al. Paracrine effect of vascular smooth muscle cells in the prevention of aortic aneurysm formation. J Vasc Surg. 2002;36:1018-26.

9. Losy F, Dai J, Pages C, Ginat M, Muscatelli-Groux B, Guinault AM, et al. Paracrine secretion of transforming growth factor-betal in aneurysm healing and 
stabilization with endovascular smooth muscle cell therapy. J Vasc Surg. 2003; 37:1301-9.

10. Franck G, Dai J, Fifre A, Ngo S, Justine C, Michineau S, et al. Reestablishment of the endothelial lining by endothelial cell therapy stabilizes experimental abdominal aortic aneurysms. Circulation. 2013;127:1877-87.

11. Hashizume R, Yamawaki-Ogata A, Ueda Y, Wagner WR, Narita Y. Mesenchymal stem cells attenuate angiotensin II-induced aortic aneurysm growth in apolipoprotein E-deficient mice. J Vasc Surg. 2011;54:1743-52.

12. Schneider F, Saucy F, de Blic R, Dai J, Mohand F, Rouard H, et al. Bone marrow mesenchymal stem cells stabilize already-formed aortic aneurysms more efficiently than vascular smooth muscle cells in a rat model. Eur J Vasc Endovasc Surg. 2013;45:666-72.

13. Fu XM, Yamawaki-Ogata A, Oshima H, Ueda Y, Usui A, Narita Y. Intravenous administration of mesenchymal stem cells prevents angiotensin II-induced aortic aneurysm formation in apolipoprotein E-deficient mouse. J Transl Med. 2013;11: 175.

14. Yamawaki-Ogata A, Fu X, Hashizume R, Fujimoto KL, Araki Y, Oshima H, et al. Therapeutic potential of bone marrow-derived mesenchymal stem cells in formed aortic aneurysms of a mouse model. Eur J Cardiothorac Surg. 2014; 45:e156-65.

15. Sharma AK, Lu G, Jester A, Johnston WF, Zhao Y, Hajzus VA, et al Experimental abdominal aortic aneurysm formation is mediated by IL-17 and attenuated by mesenchymal stem cell treatment. Circulation. 2012;126: S38-45.

16. Riera Del Moral L, Aramburu CL, Garcia JR, de Cubas LR, Garcia-Olmo D, Garcia-Arranz M. Experimental model for coadjuvant treatment with mesenchymal stem cells for aortic aneurysm. Am J Stem Cells. 2012;1:174-81.

17. Tian X, Fan J, Yu M, Zhao Y, Fang Y, Bai S, et al. Adipose stem cells promote smooth muscle cells to secrete elastin in rat abdominal aortic aneurysm. PLoS One. 2014;9:e108105.

18. Park HS, Choi GH, Hahn S, Yoo YS, Lee JY, Lee T. Potential role of vascular smooth muscle cell-like progenitor cell therapy in the suppression of experimental abdominal aortic aneurysms. Biochem Biophys Res Commun. 2013;431:326-31.

19. Kuroda Y, Kitada M, Wakao S, Nishikawa K, Tanimura Y, Makinoshima H, et al. Unique multipotent cells in adult human mesenchymal cell populations. Proc Natl Acad Sci U S A. 2010;107:8639-43.

20. Hori E, Hayakawa Y, Hayashi T, Hori S, Okamoto S, Shibata T, et al. Mobilization of pluripotent multilineage-differentiating stress-enduring cells in ischemic stroke. J Stroke Cerebrovasc Dis. 2016;25:1473-81.

21. Dezawa M. Muse cells provide the pluripotency of mesenchymal stem cells: direct contribution of Muse cells to tissue regeneration. Cell Transplant. 2016; 25:849-61.

22. Gimeno ML, Fuertes F, Barcala Tabarrozzi AE, Attorressi AI, Cucchiani R, Corrales L, et al. Pluripotent nontumorigenic adipose tissue-derived muse cells have immunomodulatory capacity mediated by transforming growth factorbeta1. Stem Cells Transl Med. 2017;6:161-73.

23. Katagiri H, Kushida Y, Nojima M, Kuroda Y, Wakao S, Ishida K, et al. A distinc subpopulation of bone marrow mesenchymal stem cells, muse cells, directly commit to the replacement of liver components. Am J Transplant. 2016;16: 468-83.

24. Alessio N, Ozcan S, Tatsumi K, Murat A, Peluso G, Dezawa M, et al. The secretome of MUSE cells contains factors that may play a role in regulation of stemness, apoptosis and immunomodulation. Cell Cycle. 2017;16: 33-44.

25. Hayase M, Kitada M, Wakao S, Itokazu Y, Nozaki K, Hashimoto N, et al Committed neural progenitor cells derived from genetically modified bone marrow stromal cells ameliorate deficits in a rat model of stroke. J Cereb Blood Flow Metab. 2009;29:1409-20.

26. Bi Y, Zhong H, Xu K, Zhang Z, Qi X, Xia Y, et al. Development of a novel rabbi model of abdominal aortic aneurysm via a combination of periaortic calcium chloride and elastase incubation. PLoS One. 2013;8:e68476.

27. Jones JA, Beck C, Barbour JR, Zavadzkas JA, Mukherjee R, Spinale FG, et al Alterations in aortic cellular constituents during thoracic aortic aneurysm development: myofibroblast-mediated vascular remodeling. Am J Pathol. 2009;175 1746-56.

28. Lu X, Dunn J, Dickinson AM, Gillespie JI, Baudouin SV. Smooth muscle alphaactin expression in endothelial cells derived from CD34+ human cord blood cells. Stem Cells Dev. 2004;13:521-7.

29. Henderson EL, Geng YJ, Sukhova GK, Whittemore AD, Knox J, Libby P. Death of smooth muscle cells and expression of mediators of apoptosis by T lymphocytes in human abdominal aortic aneurysms. Circulation. 1999, 99:96-104.

30. Diez-Tejedor E, Gutierrez-Fernandez M, Martinez-Sanchez P, RodriguezFrutos B, Ruiz-Ares G, Lara ML, et al. Reparative therapy for acute ischemic stroke with allogeneic mesenchymal stem cells from adipose tissue: a safety assessment: a phase II randomized, double-blind, placebo-controlled, singlecenter, pilot clinical trial. J Stroke Cerebrovasc Dis. 2014;23:2694-700.

31. Lewellis SW, Knaut H. Attractive guidance: how the chemokine SDF1/CXCL12 guides different cells to different locations. Semin Cell Dev Biol. 2012;23: 333-40.

32. Iseki M, Kushida Y, Wakao S, Akimoto T, Mizuma M, Motoi F, et al. Muse cells nontumorigenic pluripotent-like stem cells, have liver regeneration capacity through specific homing and cell replacement in a mouse model of liver fibrosis Cell Transplant. 2017;26:821-40.

Key Words: aortic aneurysm, stem cell therapy, Muse cell, mesenchymal stem cell 


\section{APPENDIX. SUPPLEMENTAL METHODS Muse Cell Preparation and Characterization}

Human BM MSCs were purchased from Lonza Japan (Tokyo, Japan) and used as the source of Muse and nonMuse cells. The human BM MSCs were labeled with GFP-labeled lentivirus as previously described. ${ }^{\mathrm{E} 1}$ Cells were maintained at $37^{\circ} \mathrm{C}$ and $5 \% \mathrm{CO}_{2}$ in low glucose Dulbecco's Modified Eagle's Medium (Thermo-Fisher Scientific, Waltham, Mass) containing 10\% fetal bovine serum (FBS) (Hyclone; Thermo-Fisher Scientific) and $0.1 \mathrm{mg} /$ $\mathrm{mL}$ kanamycin sulfate (Thermo-Fisher Scientific). MSCs from the fifth to eighth subcultures were used in this study. A fluorescence-activated cell sorter (Aria II, Becton Dickinson, Franklin Lakes, NJ) was used to isolate Muse and nonMuse cells from GFP-labeled human BM MSCs. Cells were first incubated with rat anti-SSEA-3 immunoglobulin (Ig)M antibody (1:500; BioLegend, San Diego, Calif) and then with allophycocyanin-conjugated goat anti-rat $\operatorname{IgM}$ antibody (1:100; Jackson ImmunoResearch, West Grove, $\mathrm{Pa}$ ). Muse cells were collected as the $\mathrm{GFP}^{+} / \mathrm{SSEA}-3^{+}$cell fraction (Supporting Information, Figure E1, P3 gate). We also collected $\mathrm{GFP}^{+} / \mathrm{SSEA}-3^{-}$cells as non-Muse cells (Supporting Information, Figure E1, P6 gate). GFP ${ }^{+}$MSCs were also sorted and used for the MSC experimental group.

A cluster formation assay in a single-cell suspension culture (for 7 days) was performed as previously described. ${ }^{\mathrm{E} 2}$ The formed clusters were individually transferred onto gelatin-coated 4-well dishes, and the cells were expanded from the adhered clusters for 14 days. Cells were fixed with 4\% paraformaldehyde in PBS and stained using the following primary antibodies: anti-neurofilament-M rabbit IgG antibody (1:200; Merck Millipore, Billerica, Mass), an ectodermal marker; anti- $\alpha$ SMA mouse IgG antibody (1:200; Thermo-Fisher Scientific), a mesodermal marker; and anti-cytokeratin 7 mouse IgG antibody (1:100; Merck Millipore), an endodermal marker. Furthermore, to identify the spontaneous endothelial differentiation ability of the clusters, the expanded cells were incubated with antiCD31 goat IgG antibody (1:50; Santa Cruz Biotechnology, Dallas, Tex). All samples were then incubated with secondary antibodies, either Alexa Fluor 488 or Alexa Fluor 568conjugated donkey anti-mouse/rabbit/goat IgG antibody (1:500; Thermo-Fisher Scientific), and imaged under laser confocal microscopy (Nikon, Tokyo, Japan).

\section{Induction of Aortic Aneurysm}

Eight-week-old male SCID mice (C.B-17/lcr-scid/scidJcl) were purchased from CLEA Japan (Tokyo, Japan). Experimental models of abdominal AA were developed by 20 minute periaortic incubation of $\mathrm{CaCl}_{2}(0.5 \mathrm{~mol} / \mathrm{L}$; Otsuka, Tokyo, Japan $)$ and porcine pancreatic elastase $(0.5 \mathrm{unit} / \mu \mathrm{L}$, Wako Pure Chemical Industries, Osaka, Japan), as previously described. ${ }^{\mathrm{E} 3}$ Briefly, the mice were anesthetized with $4.0 \%$ isoflurane using a face mask and maintained on
$1.5 \%$ to $2.0 \%$ isoflurane for the remainder of the procedure. Rectal temperature was maintained at $37^{\circ} \mathrm{C}$ using a TR-200 Heating Pad System (Fine Science Tools, British Columbia, Canada). Under microscopy (MZ6; Leica, Wetzlar, Germany), the abdominal cavity was exposed by a midline abdominal incision under sterile conditions. The aortic segment, infrarenal to the iliac bifurcation, was isolated, and lumbar arteries derived from the segment were then ligated with 11-0 nylon (Kono Seisakusho, Tokyo, Japan). The isolated region of the aorta was then circumferentially wrapped with a piece of sterile gauze that was soaked with $20 \mu \mathrm{L}$ of the $\mathrm{CaCl}_{2}$ and elastase solution and incubated for 20 minutes. The gauze was then removed, and the arterial segment was washed twice with PBS. The abdominal incision was closed with a continuous running suture. Mice incubated with saline solution for 20 minutes were used as the sham operation group (Sham Group).

\section{Total RNA Extraction, cDNA Synthesis, and Real- Time Reverse Transcriptase Polymerase Chain Reaction Analyses}

To evaluate the feasibility of using Muse cells to treat AA, we performed real-time reverse transcriptase polymerase chain reaction (PCR) of Muse cells cultured for 48 hours with $10 \%$ serum collected from SCID AA on postoperative day 3. For controls, we purchased human EPCs (BioChain, Newark, Calif) and human CD34 ${ }^{+}$progenitor cells from cord blood (PromoCell, Heidelberg, Germany). Total RNA was extracted from the all cells and purified using an RNeasy Mini Kit (QIAGEN, Venlo, Nederland). Firststrand cDNA was synthesized using SuperScript III reverse transcriptase (Invitrogen, Waltham, Mass). PCR was performed using the following predesigned primers purchased from Applied Biosystems (Thermo Fisher Scientific): forkhead box C1 (FOXC1, Hs00559473_s1), krüppel-like Factor 2 (KLF2, Hs00360439_g1), myocyte-specific enhancer factor 2C (MEF2C, Hs00231149_m1), ETS domaincontaining protein Elk-1 (ELK1, Hs00901847_m1), calcium/calmodulin-dependent protein kinase type II delta (CAMK2 $\delta$, Hs00943550_m1), myosin heavy chain 10 (MYH10, Hs00992055_m1), heat shock $70 \mathrm{kDa}$ protein A8 (HSPA8, Hs03044880_gH), protein disulfideisomerase A3 (PDIA3, Hs00607126_m1), malate dehydrogenase 1 (MDH1, Hs00936497_g1), and $\beta$-actin (ACTB, Hs01060665_g1). DNA was amplified via the Applied Biosystems 7500 real-time PCR system (Thermo-Fisher Scientific) according to the manufacturer's instructions. Data were processed using the $\Delta \Delta \mathrm{CT}$ method.

\section{Co-Culture of Muse Cells With Aortic Aneurysm Samples}

To assess the capacity of Muse cells to differentiate into vascular lineage cells, co-culture of Muse cells and AAs was performed. AA-induced SCID mice were killed by an 
overdose of isoflurane anesthesia 3 days after surgery. AAs were dissected longitudinally, and a $100-\mu \mathrm{L}$ suspension of 10,000 Muse cells was added onto the luminal side of the aorta, the side on which transfused Muse cells were considered to attach in vivo. AAs and Muse cells were co-cultured with Dulbecco's Modified Eagle's Medium (Thermo-Fisher Scientific) containing 10\% FBS (Hyclone; Thermo-Fisher Scientific) and $0.1 \mathrm{mg} / \mathrm{mL}$ kanamycin sulfate (ThermoFisher Scientific) for 1, 2, and 3 weeks, with medium exchange every 2 days. After termination of the co-culture, the aortic samples were fixed with $4 \%$ paraformaldehyde (Wako Pure Chemical Industries) in PBS, stained with 4',6-diamidino-2-phenylindole (1:1000; Sigma-Aldrich, St Louis, Mo), and then inspected using a multiphoton laser microscope (Nikon). Representative images were captured every $20 \mu \mathrm{m}$ from the luminal side to the outer side. Furthermore, the aorta was embedded in optimal cutting temperature compound (Sakura Finetek USA, Inc, Torrance, Calif) and cut sagittally using a microtome cryostat (Leica). The frozen cross-sections $(6 \mu \mathrm{m})$ were stained with anti- $\alpha$ SMA mouse IgG antibody (1:200; Thermo-Fisher Scientific), anti-nonmuscle myosin heavy chain mouse IgG antibody (1:200; YAMASA, Tokyo, Japan), anti-CD31 mouse IgG antibody (1:50; Becton Dickinson), anti-CD34 rabbit IgG antibody (1:100; Abcam, Cambridge, UK), anti-CD141 mouse IgG antibody (1:100; Becton Dickinson), and anticalponin mouse IgG antibody (1:100; DAKO, Carpinteria, Calif). All samples were incubated with secondary antibodies, either Alexa Fluor 488 or Alexa Fluor 568conjugated donkey anti-mouse/rabbit IgG antibody (1:500; Thermo-Fisher Scientific), and inspected under a laser confocal microscope (Nikon).

\section{Aortic Diameter Measurement}

The maximum transverse diameter of the AA was measured under a microscope (MC120HD, Leica) at both the initial operation and the final terminal dissection. The diameter of the AA was presented as a ratio defined as (final diameter - initial diameter)/initial diameter. We also measured the aneurysmal diameter by ultrasound inspection (S6V, SonoScape, Shenzhen, China) under general anesthesia with isoflurane on the day of the surgery, day 0 , day 7 , week 2 , week 3 , week 4 , week 5 , week 6 , week 7 , and week 8 .

\section{Specimen Preparation and Histology}

On week 3 or 8 , mice were anesthetized deeply and perfused intracardially with PBS followed by $4 \%$ paraformaldehyde in PBS. Whole abdominal aortas were harvested, divided into 5 pieces, and embedded in optimal cutting temperature compound (Sakura Finetek USA, Inc). Cross-sections $(6 \mu \mathrm{m})$ were generated using a microtome cryostat (Leica). To identify elastic fibers, ElasticaMasson staining was performed, and a BX53 microscope (Olympus, Tokyo, Japan) was used for histologic examination. Aortic medial elastin content was quantitated on the basis of the percent area of elastin compared with the total medial tissue area, which was calculated as the mean of the 5 pieces from each of the aortic samples, as described previously. ${ }^{\mathrm{E}}$ The images were quantified using the Image $\mathrm{J}$ software (v.1.41, National Institutes of Health, Bethesda, Md).

For assessment of in vivo Muse cell differentiation into VSMCs, the $6-\mu \mathrm{m}$ cryosections were double-stained with anti- $\alpha$ SMA mouse IgG antibody (1:200; Thermo-Fisher Scientific) and anti-GFP rabbit IgG antibody (1:500; Abcam), followed by secondary antibody staining with Alexa Fluor 488-conjugated donkey anti-rabbit IgG antibody (1:500, Thermo-Fisher Scientific) and Alexa Fluor 568conjugated donkey anti-mouse IgG antibody (1:500, Thermo-Fisher Scientific). Likewise, differentiation into ECs was assessed by the staining with anti-CD31 goat IgG antibody (1:50; Santa Cruz Biotechnology) and antiGFP rabbit IgG antibody (1:500; Abcam), followed by secondary antibody staining with Alexa Fluor 488-conjugated donkey anti-rabbit IgG antibody (1:500, Thermo-Fisher Scientific) and Alexa Fluor 568-conjugated donkey antigoat IgG antibody (1:500, Thermo-Fisher Scientific). The double-positive cells were quantitated by the cell number per unit total medial area and calculated as the mean of 5 pieces from each of the aortic samples.

To identify macrophages in the aortic wall in vivo, anti-F4/ 80 rat polyclonal antibody (1:100, AbDSerotec, Oxford, United Kingdom) and the secondary antibody Alexa Fluor 488-conjugated goat anti-rat IgG antibody (1:500, ThermoFisher Scientific) were used. The positive cells were quantitated as the mean of the cell number per randomly selected 5 of $0.01 \mathrm{~mm}^{2}$ regions of interest and calculated as the mean of 5 pieces from each of the aortic samples.

\section{Total DNA Extraction and Real-Time Polymerase Chain Reaction Analysis of the Human Alu Sequence}

The Muse cell (M) group and non-Muse cell (N) group mice were killed by isoflurane overdose at week 8 , and the abdominal aorta, lung, spleen, liver, heart, and kidney were harvested ( $\mathrm{n}=1$, respectively). Genomic DNA was extracted from these organs and purified using a REDExtract-N-Amp Tissue PCR Kit (Sigma-Aldrich). As reported previously, ${ }^{\mathrm{E} 5} \mathrm{PCR}$ was carried out using specific primers as follows:

(a) Forward primer, 5'-CATGGT-GAAACCCCGTCTCTA- $3^{\prime}$

(b) Reverse primer, 5'-GCCTCAGCCTCCCGAGTAG-3'

(c) TaqMan probe, 5'-FAM-ATT-AGCCGGGCGTGGTG GCG-TAMRA- $3^{\prime}$

The DNA was amplified using Applied Biosystems 7500 real-time PCR system (Thermo-Fisher Scientific) according to the manufacturer's instructions. The gene expression levels of the respective organs are presented as the ratio to 
the expression level of the abdominal aorta in the non-Muse cell $(\mathrm{N})$ group mice.

\section{Multiphoton Microscopy Aortic Samples}

To elucidate the Muse cell migration route into the aortic vessel wall, AA-induced mice were killed by perfusion fixation of $4 \%$ paraformaldehyde in PBS 3 or 5 days after the single administration of Muse cells (20,000 cells). The whole abdominal aortas were harvested and stained with $4^{\prime}, 6-$ diamidino-2-phenylindole (1:1000, Sigma-Aldrich). The aortas were then inspected using a multiphoton laser microscope (Nikon). The representative images were captured every $20 \mu \mathrm{m}$ from the outer side to the luminal side.

\section{Sample Size Calculation}

The minimum sample size $(n)$ for detecting a difference of a $150 \%$ diameter increase between any 2 groups can be calculated using $\mathrm{n}=\left(\mathrm{Z}_{\alpha}+\mathrm{Z}_{\beta}\right)^{2}(\sigma / \delta)^{2}$, where $\mathrm{Z}_{\alpha}$ is a standard approximation at a significance level of $\alpha=0.01$ to account for multiple comparison groups by Bonferroni correction $\left(\alpha\right.$ set at $\left.0.05 / 4=0.01 ; \mathrm{Z}_{\alpha}=2.57\right), \mathrm{Z}_{\beta}$ estimated at a $\beta$ error of $0.10(=1.28), \sigma$ the standard deviation of aortic diameter $\%$ increase $(\approx 100 \%), \delta$ the difference of mean aortic diameter \%increase of the group to be compared with the reference group (hypothesized to be $150 \%$ ). By using the equation, we achieved a minimum sample size of 7 mice.

\section{E-References}

E1. Hayase M, Kitada M, Wakao S, Itokazu Y, Nozaki K, Hashimoto N, et al. Committed neural progenitor cells derived from genetically modified bone marrow stromal cells ameliorate deficits in a rat model of stroke. J Cereb Blood Flow Metab. 2009;29:1409-20.

E2. Kuroda Y, Kitada M, Wakao S, Nishikawa K, Tanimura Y, Makinoshima H, et al. Unique multipotent cells in adult human mesenchymal cell populations. Proc Natl Acad Sci U S A. 2010;107:8639-43.

E3. Bi Y, Zhong H, Xu K, Zhang Z, Qi X, Xia Y, et al. Development of a novel rabbit model of abdominal aortic aneurysm via a combination of periaortic calcium chloride and elastase incubation. PLoS One. 2013;8:e68476.

E4. Jones JA, Beck C, Barbour JR, Zavadzkas JA, Mukherjee R, Spinale FG, et al. Alterations in aortic cellular constituents during thoracic aortic aneurysm development: myofibroblast-mediated vascular remodeling. Am J Pathol. 2009;175: 1746-56.

E5. McBride C, Gaupp D, Phinney DG. Quantifying levels of transplanted murine and human mesenchymal stem cells in vivo by real-time PCR. Cytotherapy. 2003;5:7-18. 

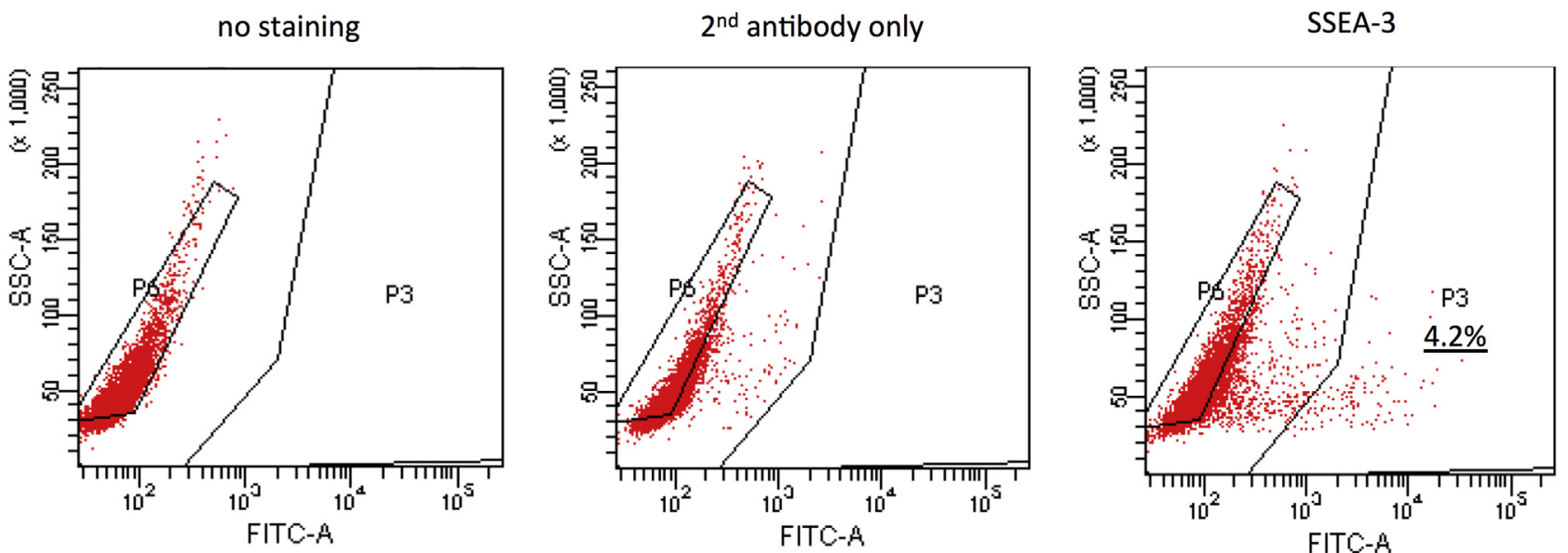

FIGURE E1. Fluorescence-activated cell sorter analysis. Gate P3 indicates SSEA- $3^{+}$cells: Muse cells. Gate P6 indicates SSEA- $3^{-}$cells: non-Muse cells. FITC-A, fluorescein isothiocyanate; SSC-A, side scatter-area.
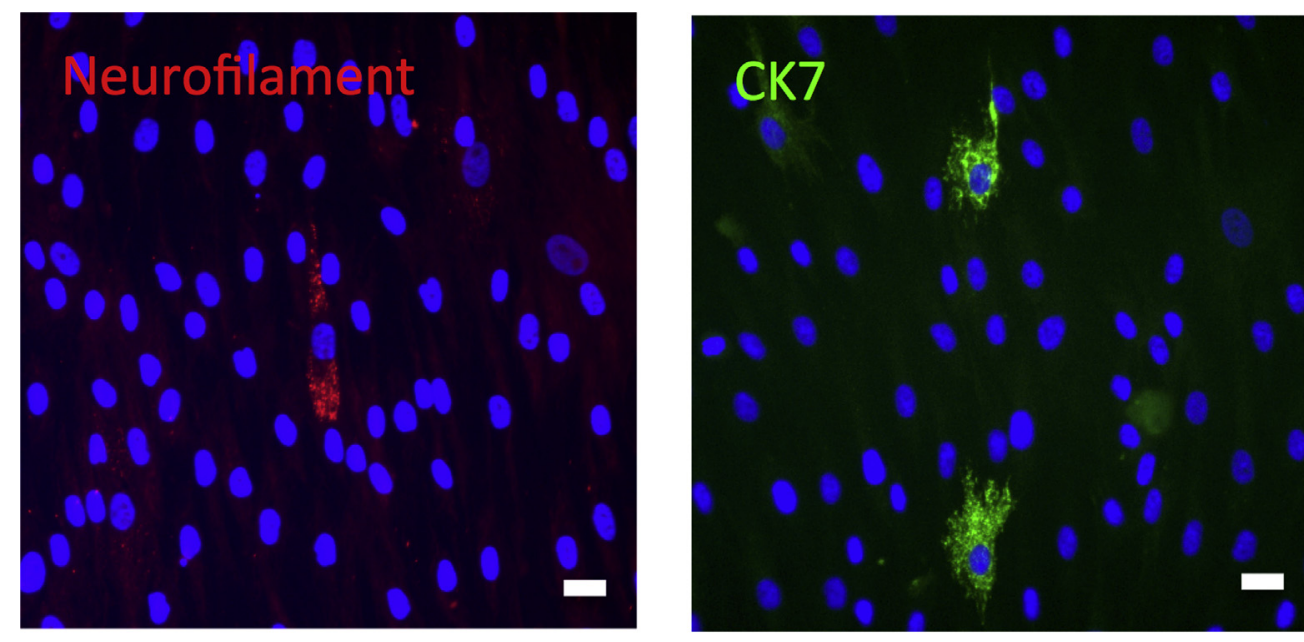

\section{Bar $=100 \mu \mathrm{m}$}

FIGURE E2. Immunostaining view of expanded cells from Muse clusters in adhesion culture media on gelatin-coated tissue culture dishes, stained with neurofilament (red, left) and cytokeratin-7 (green, right). 